\title{
LONG-DURATION RADIO TRANSIENTS LACKING OPTICAL COUNTERPARTS ARE POSSIBLY GALACTIC NEUTRON STARS
}

\author{
E. O. OfeK ${ }^{1,5}$, B. Breslauer ${ }^{2,3}$, A. Gal-Yam ${ }^{4}$, D. Frail ${ }^{3}$, M. M. Kasliwal ${ }^{1}$, S. R. Kulkarni ${ }^{1}$, And E. Waxman ${ }^{4}$ \\ ${ }^{1}$ Division of Physics, Mathematics and Astronomy, California Institute of Technology, Pasadena, CA 91125, USA \\ ${ }^{2}$ Department of Physics and Astronomy, Oberlin College, Oberlin, Ohio 44074-1088, USA \\ ${ }^{3}$ National Radio Astronomy Observatory, P.O. Box O, Socorro, NM 87801, USA \\ ${ }^{4}$ Benoziyo Center for Astrophysics, Weizmann Institute of Science, 76100 Rehovot, Israel \\ Received 2009 October 22; accepted 2010 January 21; published 2010 February 11
}

\begin{abstract}
Recently, a new class of radio transients in the $5 \mathrm{GHz}$ band and with durations of the order of hours to days, lacking any visible-light counterparts, was detected by Bower and collaborators. We present new deep near-infrared (IR) observations of the field containing these transients, and find no counterparts down to a limiting magnitude of $K=20.4$ mag. We argue that the bright ( $>1$ Jy) radio transients recently reported by Kida et al. are consistent with being additional examples of the Bower et al. transients. We refer to these groups of events as "long-duration radio transients." The main characteristics of this population are: timescales longer than 30 minutes but shorter than several days; very large rate, $\sim 10^{3} \mathrm{deg}^{-2} \mathrm{yr}^{-1}$; progenitor's sky surface density of $>60 \mathrm{deg}^{-2}$ (at $95 \%$ confidence) at Galactic latitude $\sim 40^{\circ} ; 1.4-5 \mathrm{GHz}$ spectral slopes, $f_{v} \propto v^{\alpha}$, with $\alpha \gtrsim 0$; and most notably the lack of any X-ray, visible-light, near-IR, and radio counterparts in quiescence. We discuss putative known astrophysical objects that may be related to these transients and rule out an association with many types of objects including supernovae, gamma-ray bursts, quasars, pulsars, and M-dwarf flare stars. Galactic brown dwarfs or some sort of exotic explosions in the intergalactic medium remain plausible (though speculative) options. We argue that an attractive progenitor candidate for these radio transients is the class of Galactic isolated old neutron stars (NSs). We confront this hypothesis with Monte Carlo simulations of the space distribution of old NSs, and find satisfactory agreement for the large areal density. Furthermore, the lack of quiescent counterparts is explained quite naturally. In this framework, we find: the mean distance to events in the Bower et al. sample is of order $\mathrm{kpc}$; the typical distance to the Kida et al. transients are constrained to be between $45 \mathrm{pc}$ and $2 \mathrm{kpc}$ (at the $95 \%$ confidence level); these events should repeat with a timescale of order several months; and sub-mJy level bursts should exhibit Galactic latitude dependence. We discuss two possible mechanisms giving rise to the observed radio emission: incoherent synchrotron emission and coherent emission. We speculate that if the latter is correct, the long-duration radio transients are sputtering ancient pulsars or magnetars and will exhibit pulsed emission.
\end{abstract}

Key words: brown dwarfs - galaxies: high-redshift - Galaxy: kinematics and dynamics - radio continuum: general - stars: low-mass - stars: neutron

Online-only material: color figures

\section{INTRODUCTION}

Large field-of-view radio telescope facilities such as the Parks multi-beam facility, the Arecibo multi-beam instrument, the Allen Telescope Array (DeBoer et al. 2004), and the Low Frequency Array (Falcke et al. 2007) have reinvigorated the radiofrequency time domain frontier. First signs of this "revolution" are indicated by the discoveries of new classes of radio transients. Examples include: several Galactic center sources (e.g., Hyman et al. 2005, 2009); rotating radio anomalous transients (RRATs; McLaughlin et al. 2006) which represent a previously unknown class of radio pulsars, probably twice as abundant as their "normal" cousins; and the powerful ( $\sim 30 \mathrm{Jy})$ radio "Sparker," with a timescale of several milliseconds (Lorimer et al. 2007; S. R. Kulkarni et al. 2010, in preparation).

Here, we focus on yet another emerging class of mysterious radio transients. In a novel approach, Bower et al. (2007) reanalyzed 944 epochs of Very Large Array ${ }^{6}$ (VLA) observations, taken about once per week for 22 years, of a single calibration

\footnotetext{
5 Einstein fellow.

6 The Very Large Array is operated by the National Radio Astronomy Observatory, a facility of the National Science Foundation operated under cooperative agreement by Associated Universities, Inc.
}

field. These authors discovered a total of ten transients, eight in the $5 \mathrm{GHz}$ band, and two in the $8 \mathrm{GHz}$ band. These transients can be divided into two groups: "single-epoch" and "multiepoch" transients. The eight single-epoch transients, as can be gathered by their names, were detected in only one epoch. Given a single-epoch detection, one can only constrain the duration of the transient by the epochs preceding and succeeding the time at which the transient was detected (approximately 1 week). The lower limit could be as small as the typical integration time (about 20 minutes). The two multi-epoch transients were detected after averaging over 2 months of data. Thus, the duration of these two events can be taken to be about 2 months.

Bower et al. (2007) split the data for each epoch, consisting of 20 minutes, into five segments, and looked for variability on these four minute timescales. They did not find any evidence for variability on these timescales. However, the total signalto-noise ratio $(\mathrm{S} / \mathrm{N})$ of these detections was $\lesssim 7$, and therefore the limits on variability within these 20 minute windows are appropriately weak. More importantly, the authors find the circular polarization is less than $\sim 30 \%$.

Separately, Kuniyoshi et al. (2006a, 2006b, 2007), Niinuma et al. (2007, 2009), Kida et al. (2008), and Matsumura et al. (2009) reported on a search for radio transients using an 
east-west interferometer of the Nasu Pulsar Observatory (located in Tochigi Prefecture, Japan) of Waseda University. The program consists of daily drift scanning of the sky toward the local zenith. These authors reported 11 bright radio transients (and several others were mentioned but without details), with flux density above $1 \mathrm{Jy}$ in the $1.4 \mathrm{GHz}$ band. Ten were singleepoch transients while one was detected on two successive days with flux densities of 1.7 and 3.2 Jy, respectively (Niinuma et al. 2007). In each epoch, the transients were detected for about four minutes, which is the drift scanning time, and did not exhibit any significant variation within the observation. Unfortunately, these events are not well localized and have positional uncertainties of the order of 0.4 in declination, and 0.04 in right ascension. Kida et al. (2008) stated that the $2 \sigma$ upper limit for the rate of these transients is $0.0049 \mathrm{deg}^{-2} \mathrm{yr}^{-1}$. Arguably, these events also have timescales somewhere in the range of a few minutes to a few days. Therefore, later we consider the framework in which both the VLA and the Nasu events have a common origin.

Another relevant survey was conducted by Levinson et al. (2002) who compared the NRAO VLA Sky Survey (NVSS; Condon et al. 1998) and the "Faint Images of the Radio Sky at Twenty centimeters" survey (FIRST; Becker et al. 1995; White et al. 1997). Both these surveys were undertaken in the $1.4 \mathrm{GHz}$ band and their $5 \sigma$ limits are 3.5 and $1 \mathrm{mJy}$, respectively. Levinson et al. (2002) identified nine radio transient candidates with flux densities greater than $6 \mathrm{mJy}$. Follow-up observations of these radio transients (Gal-Yam et al. 2006) showed that seven were spurious and the remaining two were plausible transients ${ }^{7}$ : an optically extinct supernova (SN) in NGC 4216 from which the radio emission lasted for several years, and VLA J172059.9+385229 (discussed in Section 2).

Bower et al. (2007) obtained deep visible-light images of their transients. They found that the multi-epoch event $\mathrm{RT}^{8} 19870422$ was $1^{\prime \prime} .5$ from a $z=0.249, R=20.2 \mathrm{mag}$ galaxy. The peak luminosity of RT 19870422 , assuming that the transient is related to this galaxy, is consistent (to an order of a magnitude) with an energetic SN similar to SN 1998bw (GRB 980425; Kulkarni et al. 1998) and SN 2006aj (Soderberg et al. 2006). We note that the rate of these events is marginally consistent with the rate of low-luminosity GRBs derived by Soderberg et al. (2006). In contrast, the other multi-epoch transient RT 20010331 has no optical counterpart to a limiting magnitude of $g \approx 27.6 \mathrm{mag}$, $R \approx 26.5 \mathrm{mag}$, and $K \approx 19.2 \mathrm{mag}$ (this paper) to within $5^{\prime \prime}$ of the radio source. The great offset between a putative host galaxy and the radio transient makes this an unusual source. We note that Cenko et al. (2008) presented an example of a GRB in a galaxy halo environment. However, only about $1 \%$ of all GRBs occurs in such environments.

The single-epoch radio transient RT 19840613 falls within the optical boundary of a $z=0.040$ spiral galaxy, but clearly lying outside the nucleus of the galaxy. On the basis of the radio luminosity (assuming association with the galaxy) and the nature of the putative host galaxy, this transient is consistent with an origin similar to that of RT 19870422 (i.e., a low-luminosity GRB).

The remaining seven single-epoch transients (one detected in $8 \mathrm{GHz}$ and six at $5 \mathrm{GHz}$ ) do not have astrometrically coincident optical, near-infrared (IR), or radio counterparts and have a point-source appearance (see Table 1). This is a major clue in

\footnotetext{
7 The term "transient" is used here in the sense that we do not detected emission in quiescence.

8 Here, RT stands for radio transient and the succeeding eight digits are yyyymmdd where yyyy is the year, $\mathrm{mm}$ is the month, and dd is the day.
}

that a large fraction of gamma-ray bursts (GRBs) and most $\mathrm{SNe}$ have detectable optical host galaxies at $R \sim 26$ mag level (e.g., Ovaldsen et al. 2007).

In order to separate the events discussed above from Sparkers (Lorimer et al. 2007; S. R. Kulkarni et al. 2010, in preparation), which have very short timescales, we refer to these events as "long-duration radio transients."

In Table 1, we summarize the observational properties of all the long-duration radio transients. We define this class as events with no optical identification and durations between hours to days. This group includes the seven known sources from Bower et al. (2007) that are not associated with any optical counterpart and have timescales shorter than about 1 week; RT 19870422 (see above); and the 11 bright transients reported by Kida et al. (2008), Niinuma et al. (2009), and Matsumura et al. (2009).

The structure of this paper is as follows. In Section 2, we reexamine the case of VLA J172059.9+385229 (Levinson et al. 2002) and show it is a spurious event. In Section 3, we present new near-IR observations of the Bower et al. field. In Section 4, we review the basic properties (areal density, annual rate) of the long-duration radio transients. Next, in Section 5 we use the observational clues to refute several plausible explanations regarding the nature of the long-duration radio transients. In Section 6 , we argue that the most attractive explanation is that these radio transients are associated with Galactic isolated old neutron stars (NSs). Finally, we discuss and summarize the results in Section 7.

\section{VLA J172059.9+385226.6: A RE-ANALYSIS}

VLA J172059.9+385226.6 was identified as a $9 \mathrm{mJy}$ source in the FIRST survey but was undetected in the NVSS (flux density, $S \lesssim 3.5 \mathrm{mJy}$ ). Each image in the FIRST survey is made from data taken several days apart (R. Becker, private communications). Searching the VLA archive, we have found this field was observed three times on 1994, August 8, 13, and 14. Re-analysis of the data shows that this source was present only in the last $10 \mathrm{~s}$ integration out of the 2.5 minute scan taken on August 8, and had 370 mJy flux density. However, a closer look at the data showed that this event was due to a previously unknown bug in the VLA recording system; this bug affected the FIRST survey. Specifically, the telescopes were repointed, but the header information was not updated. VLA J172059.9+385226.6 is, in fact, a genuine source at a different sky position $\left(\alpha=17^{\mathrm{h}} 24^{\mathrm{m}} 00^{\mathrm{s}} .50, \delta=+38^{\circ} 52^{\prime} 26^{\prime \prime} .6\right.$, J2000.0). Therefore, VLA J172059.9+385226.6 is not a real transient source. Unfortunately, follow-up visible-light Hubble Space Telescope and near-IR Keck-II observations were undertaken before this realization.

We note that Bower et al. (2007) did not find evidence for variability in their transients, within the 20 minute integration interval. Therefore, the Bower et al. transients cannot be spurious sources of the same kind (see also a detailed discussion in Bower et al. 2007). However, given the uncertain nature of other radio transients (e.g., Lorimer et al. 2007; Deneva et al. 2008), we think that some caution is warranted.

\section{NEAR-IR OBSERVATIONS OF THE BOWER ET AL. FIELD}

On UTC 2008 April 28.4, we obtained a 7500 s exposure in $K_{s}$ band of the Bower et al. field, with the Hale $5.08 \mathrm{~m}$ telescope at Palomar observatory (P200) equipped with the Wide-field IR Camera (WIRC). The field of view of WIRC contains all 
Table 1

List of Candidate Long-duration Radio Transients

\begin{tabular}{|c|c|c|c|c|c|c|c|c|c|c|c|c|c|}
\hline \multicolumn{9}{|c|}{ Transient } & \multicolumn{4}{|c|}{ Lim. mag. } & \multirow[t]{2}{*}{ Ref } \\
\hline Epoch & $\begin{array}{l}\text { R.A. } \\
\text { J2000 }\end{array}$ & $\begin{array}{l}\text { Decl. } \\
\text { J2000 }\end{array}$ & $\begin{array}{c}\text { Band } \\
(\mathrm{GHz})\end{array}$ & $\begin{array}{c}S \\
(\mu \mathrm{Jy})\end{array}$ & $\begin{array}{c}\delta t^{\mathrm{a}} \\
(\mathrm{day})\end{array}$ & $\begin{array}{l}S_{\text {next }}{ }^{b} \\
(\mu \mathrm{Jy})\end{array}$ & $\begin{array}{l}S_{\text {deep }}{ }^{c} \\
(\mu \mathrm{Jy})\end{array}$ & $S / S_{\text {deep }}$ & $\begin{array}{l}X^{d} \\
\text { cts }\end{array}$ & $\begin{array}{c}g \\
\mathrm{mag}\end{array}$ & $\begin{array}{c}R \\
\text { mag }\end{array}$ & $\begin{array}{c}K \\
\mathrm{mag}\end{array}$ & \\
\hline 19840502 & 150224.61 & +781610.1 & 5.0 & $448 \pm 74$ & 7 & $-10 \pm 68$ & $<8$ & 56 & 0.08 & 27.6 & 26.5 & 20.4 & 1 \\
\hline 19860115 & 150226.40 & +781732.4 & 5.0 & $370 \pm 67$ & 7 & $199 \pm 121$ & $<8$ & 46 & 0.07 & 27.6 & 26.5 & 20.4 & 1 \\
\hline 19860122 & 150050.15 & +781539.4 & 5.0 & $1586 \pm 248$ & 7 & $-59 \pm 164$ & $<15$ & 106 & 0.08 & 27.6 & 26.5 & 20.2 & 1 \\
\hline 19920826 & 150259.89 & +781610.8 & 5.0 & $642 \pm 101$ & 56 & $37 \pm 83$ & $<9$ & 71 & 0.08 & 27.6 & 26.5 & 19.6 & 1 \\
\hline $19970528^{\mathrm{e}}$ & 150023.55 & +781301.4 & 5.0 & $1731 \pm 232$ & 7 & $90 \pm 206$ & $<36$ & 48 & 0.07 & 27.6 & 26.5 & 20.0 & 1 \\
\hline 19990504 & 145946.42 & +782029.0 & 5.0 & $7042 \pm 963$ & 21 & $-313 \pm 1020$ & $<117$ & 60 & 0.05 & 27.6 & 26.5 & 19.2 & 1 \\
\hline 19970205 & 150129.35 & +781949.2 & 8.4 & $2234 \pm 288$ & 5 & $857 \pm 323$ & $<646$ & 3.5 & 0.08 & 27.6 & 26.5 & 20.4 & 1 \\
\hline 20050110 & 044517 & +4130 & 1.4 & $1.8 \times 10^{6}$ & 1 & $\lesssim 3 \times 10^{5}$ & $\lesssim 9 \times 10^{3}$ & 200 & 0.02 & & & & 2 \\
\hline 20050327 & 064515 & +3200 & 1.4 & $1.2 \times 10^{6}$ & 1 & $\lesssim 3 \times 10^{5}$ & ${ }^{\lesssim} 6 \times 10^{3}$ & 200 & 0.02 & & & & 2 \\
\hline 20050304 & 103943 & +3200 & 1.4 & $1.7 \times 10^{6}$ & 1 & $\lesssim 3 \times 10^{5}$ & $\lesssim 7 \times 10^{3}$ & 240 & 0.04 & & & & 2 \\
\hline 20050102 & 104306 & +4100 & 1.4 & $1.7 \times 10^{6}$ & 1 & $\lesssim 3 \times 10^{5}$ & $\lesssim 1 \times 10^{3}$ & 1700 & 0.02 & & & & 2 \\
\hline $20050213^{\mathrm{f}}$ & 144322 & +3439 & 1.4 & $3.2 \times 10^{6}$ & 1 & $\lesssim 3 \times 10^{5}$ & $\lesssim 2 \times 10^{3}$ & 1600 & 0.02 & & & & 2 \\
\hline 20040520 & 173717 & +3808 & 1.4 & $1.6 \times 10^{6}$ & 1 & $\lesssim 3 \times 10^{5}$ & $\lesssim 6 \times 10^{3}$ & 170 & 0.02 & & & & 2 \\
\hline 20050210 & 020000 & +4142 & 1.4 & $2.7 \times 10^{6}$ & 1 & $\lesssim 3 \times 10^{5}$ & $\lesssim 6 \times 10^{3}$ & 450 & 0.02 & & & & 3 \\
\hline 20051226 & 020500 & +4142 & 1.4 & $4.3 \times 10^{6}$ & 1 & $\lesssim 3 \times 10^{5}$ & $\lesssim 6 \times 10^{3}$ & 720 & 0.02 & & & & 3 \\
\hline 20061224 & 020200 & +4142 & 1.4 & $4.1 \times 10^{6}$ & 1 & $\lesssim 3 \times 10^{5}$ & $\lesssim 3 \times 10^{3}$ & 1400 & 0.02 & & & & 3 \\
\hline 20060112 & 095122 & +3300 & 1.4 & $2.2 \times 10^{6}$ & 1 & $\lesssim 3 \times 10^{5}$ & $\stackrel{\approx}{\approx} 6 \times 10^{4}$ & 40 & 0.02 & & & & 4 \\
\hline 20060118 & 103926 & +3300 & 1.4 & $1.8 \times 10^{6}$ & 1 & $\lesssim 3 \times 10^{5}$ & $\lesssim 5 \times 10^{3}$ & 360 & 0.02 & & & & 4 \\
\hline $20010331^{\mathrm{g}}$ & 150346.18 & +781541.7 & 8.4 & $697 \pm 94$ & 59 & $85 \pm 85$ & $<37$ & 19 & 0.08 & 27.6 & 26.5 & 19.2 & 1 \\
\hline
\end{tabular}

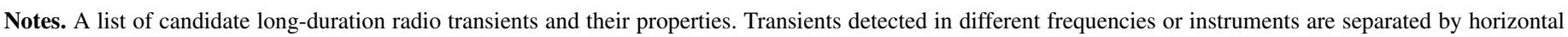

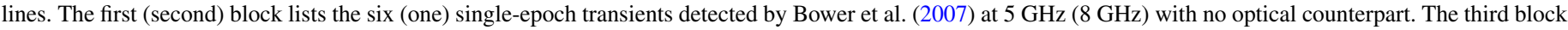

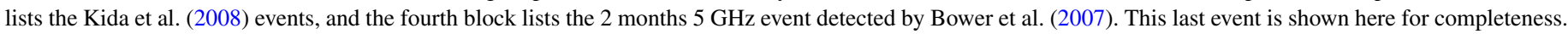

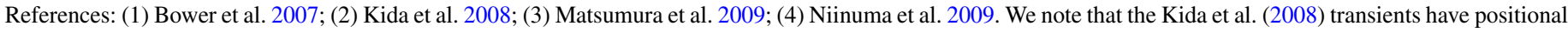
uncertainties of order 0.4 in declination, and 0.04 in right ascension.

a Time to next observation.

b Flux limit in the next observation.

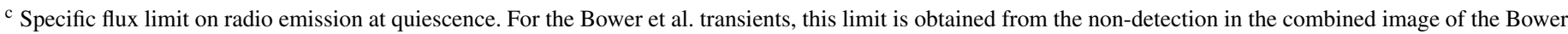

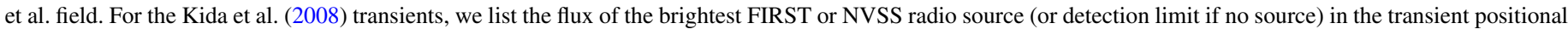
error region.

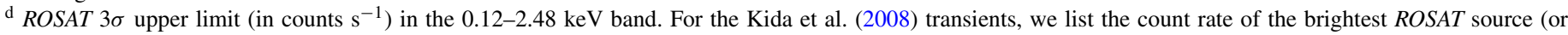

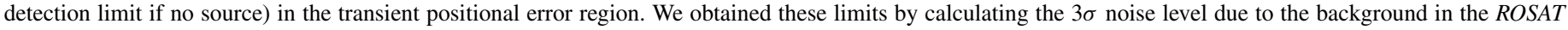
$\mathrm{X}$-ray images at the location of each transient.

e A galaxy with $R=19.6 \mathrm{mag}$ and $z=0.245,5^{\prime \prime}$ away, probably due to chance coincidence.

${ }^{\mathrm{f}}$ Detected on two epochs, separated by 1 day, with fluxes of $1.7 \mathrm{Jy}$ and $3.2 \mathrm{Jy}$ on the first and second epochs, respectively.

g RT 20011031 had a timescale of 2 months and is listed here for completeness.

the eight radio transients found by Bower et al. which do not have any visible-light counterparts. An astrometric solution was obtained using the ASCfit package (Jørgensen et al. 2002) and the images were combined using SWarp. ${ }^{9}$ Cutouts from the combined image, around the position of the eight transients, are presented in Figure 1. We do not detect any $K_{s}$-band counterparts to each of these eight transients (see Table 1).

Also listed in Table 1 are the flux limits, at the position of the transients, from the ROSAT-PSPC all-sky survey in the $0.12-2.48 \mathrm{keV}$ band (Voges et al. 1999).

\section{OBSERVATIONAL PROPERTIES OF THE LONG-DURATION RADIO TRANSIENTS}

In the following, we analyze the observational properties of the long-duration radio transients. Specifically, we discuss their rate (Section 4.1), sky surface density (Section 4.2), and source count function (Section 4.3).

\footnotetext{
9 Written by E. Bertin; http://terapix.iap.fr/
}

\subsection{Rate}

Bower et al. (2007) found that the observed areal density of events at $5 \mathrm{GHz}$ and $8 \mathrm{GHz}$ (dominated by the $5 \mathrm{GHz}$ events), with flux density above $370 \mu \mathrm{Jy}$ at a two-epoch survey, is $1.5 \pm 0.4 \mathrm{deg}^{-2}$ (based on the number of events and the effective area of the survey). Thus, the single-epoch areal density of events with flux greater than $370 \mu \mathrm{Jy}$, is $0.75_{-0.28}^{+0.40,+0.81} \mathrm{deg}^{-2}$, where the errors are given at the $1 \sigma$ and $2 \sigma$ confidence (using the formulation of Gehrels 1986). This is translated to a $5 \mathrm{GHz}$ rate of events with flux density above $370 \mu \mathrm{Jy}$ of

$$
\Re_{5 \mathrm{GHz},>0.37 \mathrm{mJy}}=540_{-200,-330}^{+290,+590}\left(\frac{t_{\text {dur }}}{0.5 \text { day }}\right)^{-1} \mathrm{deg}^{-2} \mathrm{yr}^{-1},
$$

where $t_{\text {dur }}$ is the (unknown) typical duration of these events and the errors are given at the $1 \sigma$ and $2 \sigma$ confidence. $t_{\text {dur }}$ can be a function of frequency. Therefore, comparison of this rate with rates at other frequencies should be treated with care. We are aware that our $5 \mathrm{GHz}$ rate (Equation (1)) is much larger than the rate computed by Bower et al. (2007). However, the latter estimate was the result of an arithmetic error which once corrected yields the estimate given in Equation (1). 

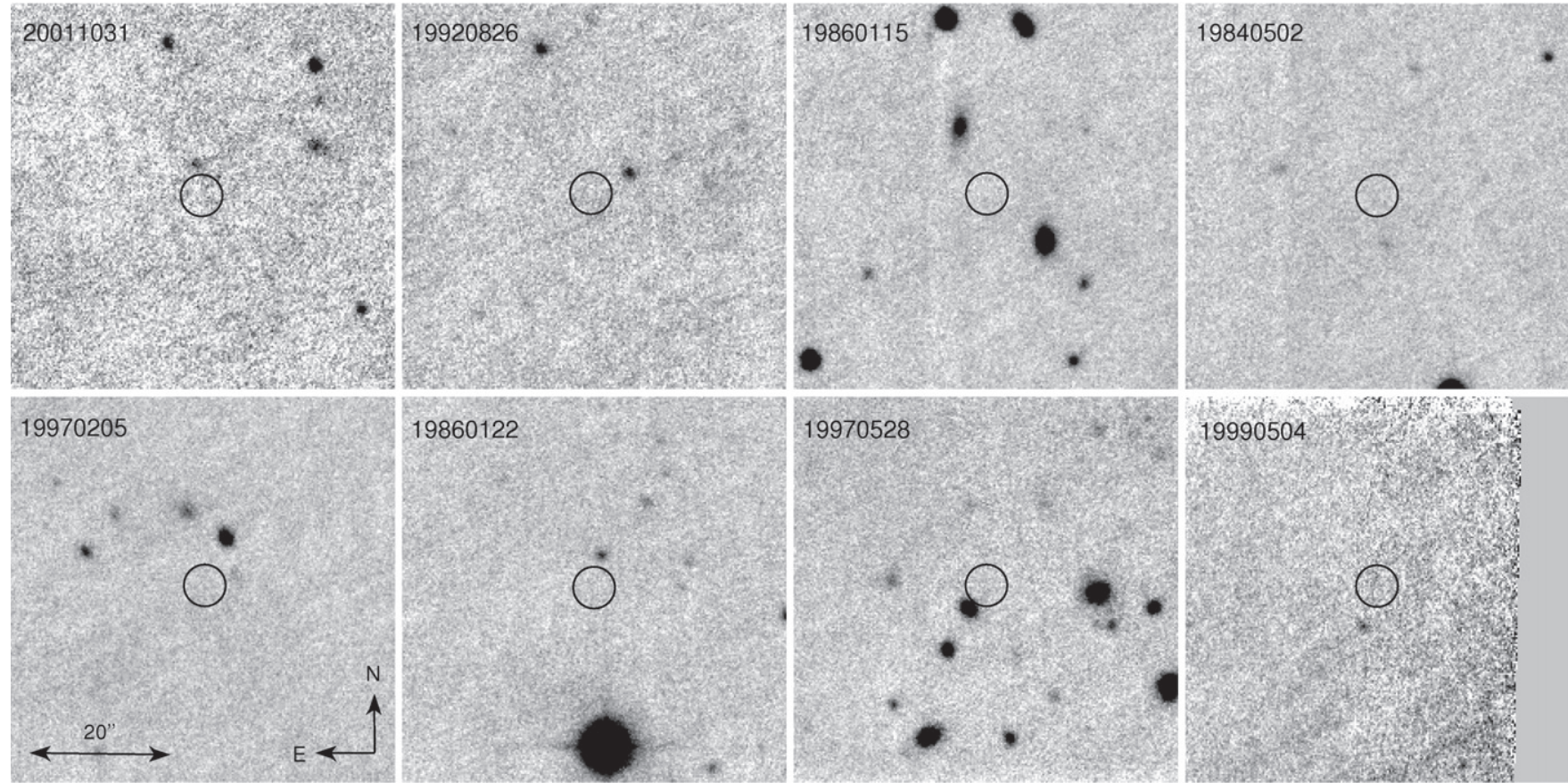

Figure 1. P200/WIRC cutouts around the locations of the eight radio transients lacking optical counterparts found by Bower et al. (2007). The position of each transient is marked by a circle with $3^{\prime \prime}$ radius. The spatial radio position uncertainty of these transients is about 0.'3 (Bower et al. 2007). The tie between the optical and radio coordinates frame is usually better than $1^{\prime \prime}$ (e.g., Gal-Yam et al. 2006). The transients' names are noted at top left of each cutout and are shown by their position east to west, upper row from left to right and than the lower row from left to right. The effective exposure times for the cutouts are $1530 \mathrm{~s}, 3030 \mathrm{~s}, 7500 \mathrm{~s}$, 7500 s, 7500 s, 5940 s, 4440 s, and 1530 s, respectively.

As can be gathered from Equation (1), the minimum rate is achieved for the largest value of $t_{\text {dur }}$ which is 7 days. Assuming a constant event rate, this minimum rate is $>9 \times 10^{15}$ (at the $95 \%$ confidence level (CL)) events over the Hubble time. For comparison, this estimate is several orders of magnitude larger than the population of any known Galactic class of stellar sources. Therefore, if long-duration radio transients are Galactic, they must be repeaters. On the other hand, if the events are catastrophic (i.e., single shot, not repeaters) then the mean time between events is $\lesssim 100 \mathrm{~s}$ (and possibly as small as $\sim 1 \mathrm{~s}$ ). The only known cosmological population with such high rate is $\mathrm{SNe}\left(\sim 1 \mathrm{~s}^{-1}\right.$; see Section 5.1).

Next we look into the rates of these events in the $1.4 \mathrm{GHz}$ band. As noted earlier (Section 2), the FIRST-NVSS analysis did not result in a firm detection of any long-duration transient. The total survey area of the FIRST-NVSS search, after correcting it for point-source incompleteness, source confusion due to the poor resolution of the NVSS, and missing NVSS data, is $2500 \mathrm{deg}^{2}$ (see Levinson et al. 2002 for details). We place an upper limit to the sky density (i.e., density of sources observed in a single epoch) of transient sources (flux density above $6 \mathrm{mJy}$ in the $1.4 \mathrm{GHz}$ band) of $1.5 \times 10^{-3} \mathrm{deg}^{-2}$ and $2.6 \times 10^{-3} \mathrm{deg}^{-2}$, at the $95 \%$ and $99.73 \% \mathrm{CL}$, respectively. We note that this sky density is consistent with the upper limit derived by Carilli et al. (2003). Therefore, the $95 \%$ confidence upper limit on the $1.4 \mathrm{GHz}$ rate, $\Re$, of long-duration radio transients with flux density above $6 \mathrm{mJy}$ is

$$
\Re_{1.4 \mathrm{GHz},>6 \mathrm{mJy}}<1.1\left(\frac{t_{\mathrm{dur}}}{0.5 \text { day }}\right)^{-1} \mathrm{deg}^{-2} \mathrm{yr}^{-1} .
$$

\subsection{Sky Surface Density}

Another interesting quantity is the areal density of the transients, $\Sigma$. We obtain a lower limit by dividing the number of unique sources by the angular area of the VLA field. All the Bower et al. transients were found within $9^{\prime}$ (twice the halfpower radius at $5 \mathrm{GHz}$ ) from the center of a single VLA field $\left(\alpha=15^{\mathrm{h}} 02^{\mathrm{m}} 20^{\mathrm{s}} .53, \delta=+78^{\circ} 16^{\prime} 14^{\prime \prime} .905 ; \mathrm{J} 2000.0\right)$. Within the half-power radius of 4.5 (corresponding to a solid angle of $0.018 \mathrm{deg}^{2}$ ) there are four detections (see Figure 2 in Bower et al. 2007). At $95 \%$ CL, the smallest number of sources is $>1.1$ (using the formulation of Gehrels 1986). Therefore, at Galactic latitude $b \sim 40^{\circ}, \Sigma>60 \mathrm{deg}^{-2}$ (95\% CL).

We note that the lack of any repeater events in the Bower et al. (2007) sample can be used to improve this limit. This can be done by calculating the probability of choosing seven events out of $N$ with no repetition. However, the resulting areal density is only increased by $40 \%$ (to the same $95 \% \mathrm{CL}$ ). We therefore retain the simpler estimate.

Assuming a constant surface density as a function of Galactic latitude the all-sky surface density of radio transient progenitors is $\Sigma_{\text {all-sky }}>3.5 \times 10^{6}$. We note that if the long-duration radio transients are Galactic then their sky surface density toward the Galactic plane should be higher, and therefore the total all-sky number could be larger.

\subsection{Source Number Count Function}

The source number count function, $N(>S)$, where $N$ is the number of events brighter than a peak flux density $S$, may provide some hints regarding the nature of the radio transient population. We consider a power-law source count function, $N(>S) \propto S^{n}$, where $n$ is the power-law index. For a homogeneous population of sources residing in an Euclidean universe we expect $n=-3 / 2$, while for Galactic thin disk population $n \approx-1$.

Assuming that $t_{\text {dur }}$ does not depend on the frequency, we can use the transient rates given in Section 4.1 to put limits on the power-law index, $n$, of the source number count function. For 


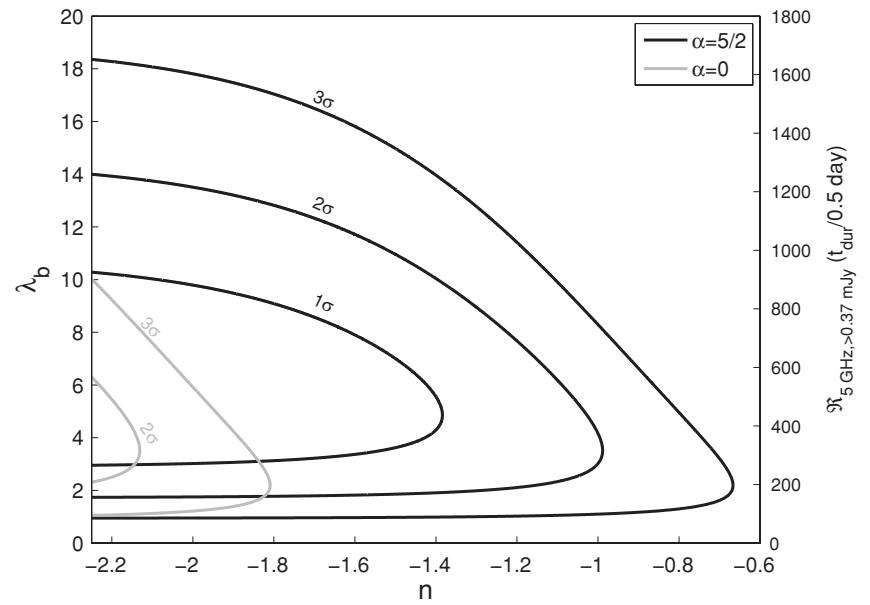

Figure 2. Confidence interval contours (as calculated from the log-likelihood) as a function of the expected value of the number of events in the Bower et al. (2007) search, $\lambda_{b}$, and the power-law index $n$, of the source number count function (see Equation (3)). The gray lines show the $2 \sigma$ and $3 \sigma$ contours for spectral index $\alpha=0$, while the black lines show the $1 \sigma, 2 \sigma$, and $3 \sigma$ contours for $\alpha=5 / 2$. The right-hand $y$-axis is the corresponding transients rate assuming $t_{\text {dur }}=0.5$ day.

each expected value of the number of events detected in the Bower et al. (2007) survey, $\lambda_{b}$, and the Levinson et al. (2002) search, $\lambda_{l}$, we calculate the probability that $k_{b}=6$ events will be detected in the Bower et al. search and $k_{l}=0$ in the Levinson et al. survey:

$$
P=\frac{1}{k_{l} ! k_{b} !} \lambda_{b}^{k_{b}} e^{-\lambda_{b}} \lambda_{l}^{k_{l}} e^{-\lambda_{l}}
$$

The power-law index, $n$ relates to $\lambda_{b} / \lambda_{l}$ through:

$$
\frac{\lambda_{b}}{\lambda_{l}}=\frac{A_{b}}{A_{l}}\left(\frac{v_{b}}{v_{l}}\right)^{n}\left(\frac{S_{b}}{S_{l}}\right)^{\alpha},
$$

where $A_{b}=9.33 \mathrm{deg}^{2}$ is the effective ${ }^{10}$ (single epoch) search area of the Bower et al. survey. This was estimated by dividing the number of transients found by Bower et al. (7), by the one epoch search areal density $\left(0.75 \mathrm{deg}^{-2}\right) . A_{l}=2500 \mathrm{deg}^{2}$ is the area of the Levinson et al. search, $v_{b}=5 \mathrm{GHz}$ and $v_{l}=1.4 \mathrm{GHz}$ are the surveys' frequencies, $S_{b}=0.37 \mathrm{mJy}$ and $S_{l}=6 \mathrm{mJy}$ are their specific flux limits, and $\alpha$ is the spectral power-law index of the sources $\left(S_{v} \propto v^{\alpha}\right)$. Equation (4) contains three parameters: $n, \lambda_{l}$, and $\lambda_{b}$. However, $\lambda_{l}$ is a function of $n$ and $\lambda_{b}$. Thus, we need only explore the phase space of two of these (e.g., $n$ and $\left.\lambda_{b}\right)$.

We calculated the probability in Equation (3) as a function of $n$ and $\lambda_{b}$. In Figure 2, we show the log-likelihood contours as a function of $n$ and $\lambda_{b}$ for $\alpha=5 / 2$ and for $\alpha=0$. The contours are for the $1 \sigma, 2 \sigma$, and $3 \sigma$, assuming two degrees of freedom (e.g., Press et al. 1992).

For $\alpha=0$, we find $n<-1.8$ at the $3 \sigma$ CL. Since such a steep number count index is unlikely for astrophysical sources, we conclude that most probably $\alpha \gtrsim 0$. The highest $\alpha$ possible for continuum emission is $\alpha=5 / 2$ (synchrotron self-absorption; Rybicki \& Lightman 1979, p. 186). For such $\alpha$, we find that $n<-0.7(-1.0)$ at the $3(2)-\sigma$ CL.

There are at least two caveats in our analysis. First, we assume that the source luminosity function does not depend on distance. 10 This is the area of a single-epoch observation multiplied by the number of
epochs.
Second, the Levinson et al. (2002) and Bower et al. (2007) rates were measured at different celestial positions. Therefore, if the long-duration radio transients are Galactic sources, then their sky distribution is probably not uniform, and this may affect the results presented in this section (however, see discussion in Section 6.1).

\section{THE PROGENITORS OF LONG-DURATION RADIO TRANSIENTS}

In this section, we list astrophysical sources and phenomenon that may be responsible for the long-duration radio transients. Some of these possibilities were already presented by Bower et al. (2007). We first discuss the extragalactic hypothesis (Section 5.1) followed by Galactic progenitors (Section 5.2).

\subsection{Extragalactic Sources}

When observing the error boxes of known types of extragalactic explosions (e.g., GRBs and $\mathrm{SNe}$ ), we usually detect the host galaxy (e.g., Fruchter et al. 2006; Ovaldsen et al. 2007; Perley et al. 2009). In Figure 3, we present a histogram of the $R$-band magnitude (or limiting magnitude) for host galaxies of a sample of Swift (Gehrels et al. 2004) detected GRBs (Ovaldsen et al. 2007). A high fraction $(\sim 50 \%)$ of the Swift GRBs are associated with galaxies brighter than about $R \sim 25$ mag. For optically identified SNe in blind surveys, the fraction is almost $100 \%$. We note that we still do not have deep images, and therefore constraints on the hosts, of the new class of bright $\mathrm{SNe}$ (Barbary et al. 2009; Quimby et al. 2009).

We find it significant that only one of the Bower et al. transients has an optical counterpart (see Section 1). It is furthermore curious that this counterpart is a low-redshift galaxy (RT 19840613; $z \cong 0.04$ ). Note the absence of any intermediate redshift counterparts.

Another way to quantify this curious absence of host galaxies is by using the observed star formation rate in the universe. In Figure 4, we show the probability that the minimum redshift in a sample of seven sources randomly selected from the observed star formation rate distribution in the Universe (Hopkins \& Beacom 2006) will be smaller than a given redshift. From Figure 4, we conclude that at least one of the seven radio transients will have $z<1.5$, at the $99.73 \% \mathrm{CL}$. The luminosity function of galaxies at high redshift is not well known. However, the GRB host galaxies sample of Ovaldsen et al. (2007; Figure 3) which probe typical redshifts of $\gtrsim 2$ suggest that if the radio transients reside in hosts similar to those of GRBs, then Bower et al. should have detected optical counterparts to most of those transients.

The above discussion notwithstanding we now consider the usual suspects in the extragalactic framework.

$G R B s$. GRBs are often detected in radio frequencies for periods of days to weeks (e.g., Frail et al. 1997; Chandra et al. 2008). However, as graphically demonstrated by Figure 3 the GRB hypothesis can be excluded. Furthermore, the observed all-sky rate of GRBs is 2 day $^{-1}$, while the all-sky rate of long-duration radio transients is $\gtrsim 10^{3}$ day $^{-1}$ (Section 4.1).

Orphan GRBs. Observational evidences suggest that GRB emission is beamed and highly anisotropic (e.g., Harrison et al. 1999; Levinson et al. 2002). Therefore, the actual rate of orphan GRB explosions is $f_{b}^{-1}$ times larger than the observed rate. Here, $f_{b}^{-1}$ is the inverse of the beaming factor, and it is probably in the range 50-500 (e.g., Guetta et al. 2005; Gal-Yam et al. 2006). However, orphan GRB radio afterglows are expected to have 


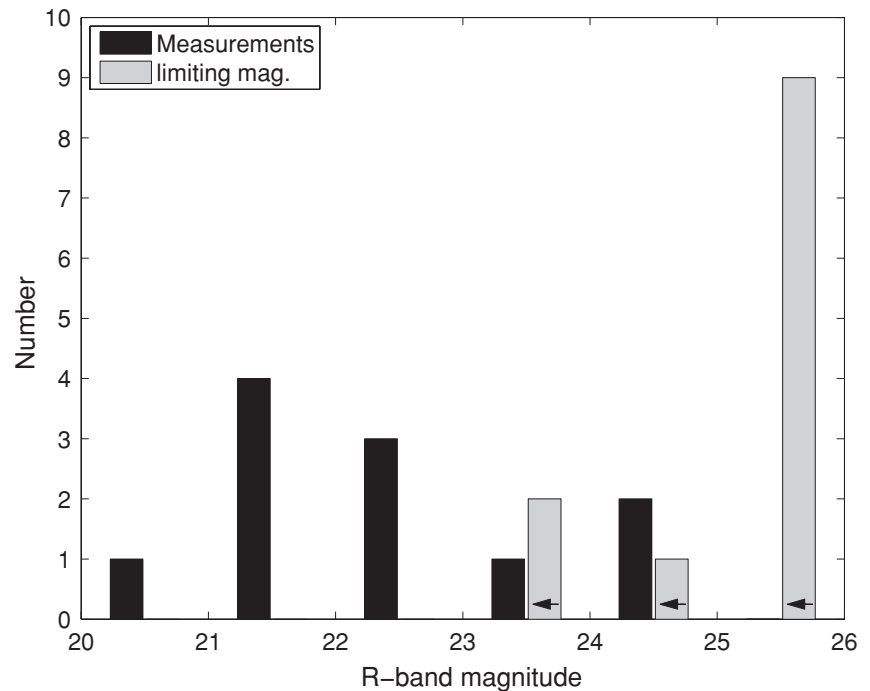

Figure 3. $R$-band magnitude (black bars) or $2 \sigma$ limiting magnitude (gray bars) distribution of GRB host galaxies (Ovaldsen et al. 2007). In some cases, the observations were done in a different band than $R$ band; in these cases, we converted the host galaxy's magnitude to $R$ band by assuming it is an Sc-type galaxy at redshift of 2, based on the galaxy spectral templates of Kinney et al. (1996).

timescales of years rather than days, which is not in line with the timescales of long-duration radio transients. More importantly, such orphans are expected to be at redshifts lower than that of GRBs (which are beamed and thus seen at higher redshift) making the host galaxy non-detections even more problematic (see Levinson et al. 2002).

Quasars and active galactic nuclei. To a limiting magnitude of $i \approx 26 \mathrm{mag}$, the faintest quasars ${ }^{11}$ can be detected to a redshift of about 5. The lack of optical counterparts associated with these transients is not consistent with a quasar connection. Low-luminosity quasars (i.e., active galactic nuclei (AGNs)), are fainter, but if their abundance roughly follows the star formation rate in the universe, then, as shown in Figure 4, the host galaxies should have been found in visible light.

Obscured quasars, also known as type-II quasars are faint in visible-light frequencies. However, these sources may reveal themselves in near-IR (e.g., Gregg et al. 2002; Reyes et al. 2008). Figure 5 shows the $g-K$ and $R-K$ color of "normal" quasars as a function of redshift. The minimum $g-K$ color of quasars is about $2.5 \mathrm{mag}$. The non-detection of AGNs down to $K_{s}$-band magnitude of 20.4 (Section 3) corresponds to a non-detection of a quasar with intrinsic $g$-band magnitude fainter than 23 . This is based on the assumption that type-II quasars and normal quasars have similar $K$-band luminosity functions.

To this magnitude level, the faintest quasars can be detected up to $z \approx 2$. Therefore, the fact that we do not detect any nearIR sources associated with the long-duration radio transients disfavors association with reddened quasars.

Supernovae. There are two known variants of bright radio SNe: type-II radio SNe, for which SN 1979C (Weiler et al. 1991) is the prototype, and type-Ic radio SN (e.g., SN 1998bw; Kulkarni et al. 1998).

Type-II radio $\mathrm{SNe}$ have long timescales (years) and are detectable in nearby galaxies $(z \sim 0.01)$. On the other hand, type-Ic radio $\mathrm{SNe}$ are detected to somewhat larger distances

\footnotetext{
11 The taxonomic definition of quasars is nuclear absolute magnitude $M_{B}<-21.5+5 \log _{10} h_{0}$, where $h_{0}$ is the present day Hubble parameter in units of $100 \mathrm{~km} \mathrm{~s}^{-1} \mathrm{Mpc}^{-1}$ (Peterson 1997).
}

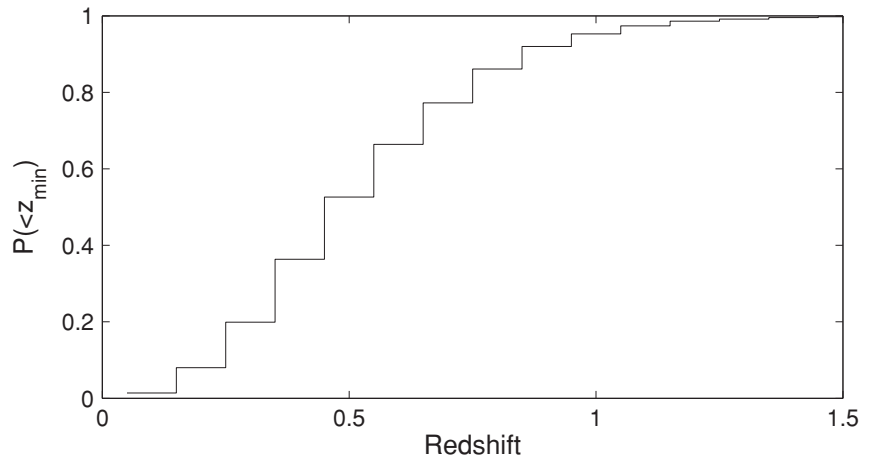

Figure 4. Cumulative probability that the minimum redshift out of seven sources, randomly selected from the star formation distribution, will be smaller than a given redshift. We used the star formation distribution compiled by Hopkins \& Beacom (2006) using the parameterization of Cole et al. (2001) and assuming a modified Salpeter IMF. We divided this star formation distribution by $(1+z)$ to account for the decreasing transients rate due to time dilation.

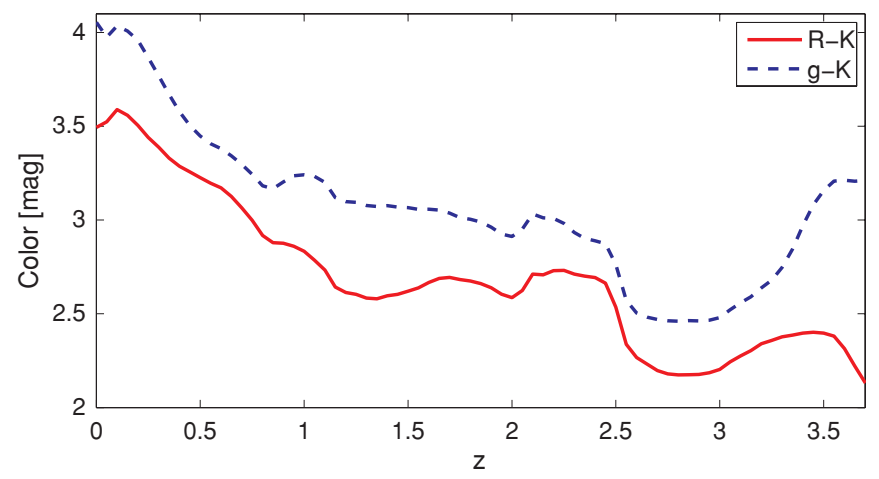

Figure 5. $R_{\text {Vega }}-K_{\text {Vega }}$ (solid line), and $g_{\mathrm{AB}}-K_{\text {Vega }}$ (dashed line) colors, of quasars, as a function of redshift $z$. The colors are based on synthetic photometry of quasar spectral templates (Brotherton et al. 2001; Glikman et al. 2006). Synthetic photometry is calculated using the code of Poznanski et al. (2002). The minimum $g-K$ in the range $z=0$ to $z=3.7$ is about $2.5 \mathrm{mag}$. However, at $z>3.7$ the $g-K$ color will be larger since the Ly $\alpha$ line is found at wavelength redder than that of the $g$ band.

(A color version of this figure is available in the online journal.)

$(z \sim 0.1)$ and last a few weeks. Therefore, based on the lack of optical counterparts we can rule out association with type-II or type-Ic radio $\mathrm{SNe}$.

Extragalactic microlensing. When considering microlensing events we should discuss the population of sources and lenses. While the lenses can be "unseen" objects, the sources should be radiant. Most sub-mJy sources, which are the potential sources for microlensing, are starburst galaxies. These galaxies have spatial size several orders of magnitude larger than the Einstein radius of stellar-mass lenses. Therefore, microlensing by extragalactic or Galactic objects cannot explain the high amplitude of the radio transients discussed here (Table 1).

We note that the small number of background sources and the high amplitude of the transients also rule out scintillation events.

Extragalactic soft gamma-ray repeaters (SGRs). There are only eight ${ }^{12}$ SGRs known in the Milky Way galaxy and the Magellanic Clouds. However, extragalactic SGRs (e.g., Eichler 2002; Nakar et al. 2006; Popov \& Stern 2006; Ofek et al. 2006, 2008; Ofek 2007) may be detected to larger distances. Currently, the radio emission from giant flares of SGRs is

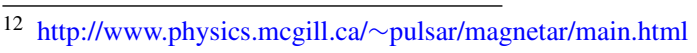


not well constrained (see Cameron et al. 2005). Assuming a fraction of $10^{-4}$ of the energy released in hard X-rays $/ \gamma$-rays is emitted in radio over $1 \mathrm{hr}$, a giant X-ray flare with luminosity $L_{X} \sim 10^{46} \mathrm{erg}$ can be observed to a distance of a about $100 \mathrm{Mpc}$. However, the rate of SGR giant flares with such energy is about $10^{-4}-10^{-5} \mathrm{Mpc}^{-3} \mathrm{yr}^{-1}$ (Ofek 2007). Therefore, the expected observed rate of Galactic and extragalactic SGRs is at least 3 orders of magnitude smaller than the rate of long-duration radio transients.

New unknown explosions. Explosions taking place outside galaxies ("naked") may explain the lack of optical counterparts. In general, we cannot rule out putative extra-Galactic sources, which have not been discovered yet.

We discuss several examples of such putative sources, showing that they are unlikely sources for the long-duration radio transients. Hawking (1974) suggested that primordial black holes of mass $\lesssim 10^{15} \mathrm{~g}$ will evaporate within the Hubble time, and eventually emit a burst of energetic photons and particles. Such explosions are expected to manifest as a short-duration $(\ll 1 \mathrm{~s})$ radio pulse as the ambient magnetic field is altered by an expanding conducting shell (Rees 1977). However, to date such events were not found (e.g., Phinney \& Taylor 1979). Moreover, they are expected to have very short timescales, in contrary to long-duration radio transients.

Following the suggestion by S. R. Kulkarni et al. (2010, in preparation), Vachaspati (2008) presented a model in which grand unification scale superconducting cosmic strings are emitting short $(<1 \mathrm{~s})$ radio flares. Vachaspati (2008) predicts that the source number count function of such events will be $N(>S) \propto S^{-1 / 2}$, which is not consistent with the long-duration radio transients source count function (Section 4.3).

Strong radio emission from $\mathrm{SNe}$ was suggested by Colgate $\&$ Noerdlinger (1971) and Colgate (1975). In their scenario, the expanding core of the SN "combs" the star's intrinsic dipole field, and the generated current sheet produces coherent radio emission. The maximum energy emitted in such a radio pulse, assuming no attenuation, is of the order of $10^{46} \mathrm{erg}$. However, the expected pulse is very short $(\ll 1 s)$ in comparison to the observed timescale of long-duration radio transients. Of course, the lack of detectable host galaxies makes any such suggestion untenable.

The lack of optical counterparts cannot rule out association with high-redshift sources (e.g., Population-III stars). Assuming a large cosmological distance, the rest-frame energy of such events is approximately

$$
\begin{aligned}
E_{f, \cos } \approx & 3 \times 10^{48} \frac{S_{v}}{0.4 \mathrm{mJy}} \frac{\Delta v}{10^{10} \mathrm{~Hz}} \frac{t_{\mathrm{dur}}}{0.5 \mathrm{day}}\left(\frac{d_{L}}{5 \times 10^{10} \mathrm{pc}}\right)^{2} \\
& \times\left(\frac{1+z}{6}\right) \mathrm{erg} ;
\end{aligned}
$$

here, $d_{L}$ is the luminosity distance (normalized to $1+z=6$ ), $z$ is the redshift, and $S_{\nu}, \Delta v$, and $t_{\text {dur }}$ are given in the observed frame.

The rest-frame brightness temperature (normalize at $1+z=6$ ) of such events is

$$
\begin{aligned}
T_{B, \cos }= & S_{v, \text { rest }} d_{\text {lum }}^{2}\left(c / v_{\text {rest }}\right)^{2}\left(2 \pi k_{B} R_{\text {rest }}^{2}\right)^{-1} \\
\approx & 7 \times 10^{16} \frac{S_{v}}{0.4 \mathrm{mJy}}\left(\frac{1+z}{6}\right)^{-1}\left(\frac{d_{L}}{5 \times 10^{10} \mathrm{pc}}\right)^{2} \\
& \times\left(\frac{v}{5 \mathrm{GHz}}\right)^{-2}\left(\frac{R_{\text {rest }}}{200 \mathrm{au}}\right)^{-2} \mathrm{~K},
\end{aligned}
$$

where we set the size of the emission region, $R_{\text {rest }}$, to 200 au which is the light crossing time in the rest frame, $c t_{\text {dur,rest }}=$ $c t_{\text {dur }}(1+z)^{-1}$, for $t_{\text {dur }}=7$ day. Again, $S_{v}, \Delta v$, and $t_{\text {dur }}$ are given in the observer frame, while the subscript "rest" indicates quantities at the rest frame. As $t_{\text {dur }} \lesssim 7$ day, this is a lower limit on the brightness temperature. The huge brightness temperature, relative to the minimum energy or equipartition temperature (Readhead 1994), requires a coherent emission mechanism or alternatively a Lorentz factor of $\gtrsim 10^{2}$. However, if the beaming factor is large, then the rate of long-duration radio transients will exceed the $\mathrm{SN}$ rate in the universe.

\subsection{Galactic}

Stellar sources. Several types of Galactic sources which are known to flare at radio wave bands were discussed by Bower et al. (2007). Specifically, RS CVn stars, FK Com stars, Algol class binaries, and X-ray binaries are ruled out by the lack of optical counterparts. Other possibilities, like T Tau stars, are associated with star-forming regions typically found at low Galactic latitude.

Late-type stars are known to flare in radio wave bands (see Güdel 2002 for review). However, the lack of $R$ - and $K$-band optical/near-IR counterparts rules out an association with latetype $\mathrm{M}$ dwarfs up to a distance of $2 \mathrm{kpc}$, which is the median distance of Galactic M dwarfs at the direction of the Bower et al. field (Gould et al. 1996). Moreover, flares from M dwarfs (and brown dwarfs) are known to exhibit strong circular polarization ( 100\%; e.g., Hallinan et al. 2007; Berger 2006). However, as summarized in Section 1, Bower et al. (2007) did not find evidence for circular polarization above $30 \%$ in any of their radio transients.

Brown dwarfs. In recent years, it was found that at least some brown dwarfs are active, and show bursts in radio and X-ray wave bands (e.g., Burgasser et al. 2000; Berger et al. 2001; Berger 2002; Burgasser \& Putman 2005). In the radio regime, these flares peak around $5 \mathrm{GHz}$ (spectral slopes $\alpha \gtrsim 0$; Berger et al. 2001, 2005, 2008a, 2008b).

Brown dwarfs are potentially very faint sources. Our near-IR search of the Bower et al. (2007) field can detect a T5-type brown dwarf up to distances of about $250 \mathrm{pc}$. However, our observations cannot rule out an association of the Bower et al. transients with older, or less massive, and hence cooler and fainter brown dwarfs (e.g., Y-class brown dwarfs; see Burrows et al. 1997; Baraffe et al. 2003 for evolutionary models).

For cooler brown dwarfs which are undetectable by our $K$ band search, based on the minimum surface density of radio transient progenitors derived in Section 4.2, we can put a rough lower limit on the distance. We assume that the local density of brown dwarfs with effective temperature above $200 \mathrm{~K}$ is $\rho_{\mathrm{BD}} \approx 0.1 \mathrm{pc}^{-3}$ (Reid et al. 1999; Burgasser 2004) and that at small distances the distribution can be regarded as isotropic. Agreement between the areal density of the radio transients (Section 4.2) to that of old brown dwarfs is obtained by having the brown dwarfs at a typical distance of $d \gtrsim 200$ pc.

At such distances, the energy needed to produce a single long-duration radio transient burst will be about

$$
\begin{aligned}
E_{\mathrm{f}, \mathrm{BD}} \approx & 8 \times 10^{30} \frac{S_{v}}{0.4 \mathrm{mJy}}\left(\frac{d}{200 \mathrm{pc}}\right)^{2} \frac{\Delta v}{10^{10} \mathrm{~Hz}} \\
& \times \frac{t_{\text {dur }}}{0.5 \text { day }} \mathrm{erg} .
\end{aligned}
$$


Should the Bower et al. radio transients arise from brown dwarfs then they must repeat. The flare repetition timescale is $\Sigma / \Re$. For the minimum sky surface density of $\Sigma \sim 60 \mathrm{deg}^{-2}$ (Section 4.2), and assuming a rate $\Re \approx 500 \mathrm{yr}^{-1} \mathrm{deg}^{-2}$, the repetition timescale is $\gtrsim 50$ day.

For a typical distance of $200 \mathrm{pc}$, the total energy emitted over the Hubble time from a single active brown dwarf will be $\sim 10^{42}$ erg. For comparison, the magnetic energy of active late-type $\mathrm{M}$ dwarfs is

$$
\begin{aligned}
E_{B} & =\frac{4}{3} \pi R_{*}^{3} \frac{B^{2}}{8 \pi} \\
& \cong 5.1 \times 10^{35}\left(\frac{r_{*}}{70,000 \mathrm{~km}}\right)^{3}\left(\frac{B}{3 \mathrm{kG}}\right)^{2} \mathrm{erg},
\end{aligned}
$$

where $R_{*}$ is the star radius and $B$ is its magnetic field. We note that magnetic fields in the most magnetically active $M$ dwarfs reach several kG (e.g., Valenti et al. 1995; Johns-Krull \& Valenti 1996; Berger et al. 2008b). This is several orders of magnitude smaller than the total energy output of a longduration radio transient. Therefore, this scenario requires that the magnetic field of brown dwarfs will be replenished from an internal energy source. This is not an implausible suggestion. For example, $10^{42} \mathrm{erg}$ is only a small fraction of the brown dwarf thermal or rotational energy. The strongest argument against the association of long-duration radio transients with brown dwarfs is the lack of circular polarization observed in the Bower et al. (2007) events.

Reflected solar flares. As discussed by Bower et al. (2007), a solar flare reflected by a solar system object will have a flux of

$$
\begin{aligned}
S_{\text {ref }}= & 6.6 \frac{S_{\odot, \text { flare }}}{10^{8} \mathrm{Jy}} \frac{A}{0.6}\left(\frac{d}{100 \mathrm{~km}}\right)^{2} \\
& \times\left(\frac{r}{1 \mathrm{au}}\right)^{-2}\left(\frac{\Delta}{1 \mathrm{au}}\right)^{-2} \mu \mathrm{Jy},
\end{aligned}
$$

where $S_{\odot, \text { flare }}$ is the solar flare flux density, $r$ and $\Delta$ are the distance of the asteroid from the Sun and Earth, respectively, and $A$ is its albedo. ${ }^{13}$ Strong $\mathrm{X}$-class solar flares may have flux densities of $10^{8} \mathrm{Jy}$, although they typically last less than 20 minutes (Bastian et al. 1998). Moreover, in order to explain the long-duration radio transients using such reflected events, we need a population of huge asteroids $(d>100 \mathrm{~km})$, at near Earth orbits, which do not exist. More importantly, the motion of such objects will be detectable by the VLA in A array whereas the Bower et al. transients are point sources.

Pulsars. Most of the Galactic pulsars are young objects (up to $10^{8} \mathrm{yr}$ ), and found near the Galactic plane. Moreover, with the exception of magnetars, known pulsars have radio spectrum with $\alpha \approx-2$ (e.g., Camilo et al. 2006). Based on the catalog of simulated NSs (Ofek 2009), the expected surface density of NSs with age smaller than 10 (100) Myr, at the direction of the Bower et al. field, is about $0.3(8) \mathrm{deg}^{-2}$, assuming $10^{9} \mathrm{NSs}$ in the Galaxy. We note that the number of NSs in the direction of the Bower et al. field ( $b \cong 37^{\circ}$ ) rises faster than linearly with age, since NSs are born in the Galactic plane and ejected (mostly) to high Galactic latitudes.

Isolated old neutron stars. The Galaxy may host about $10^{9}$ old isolated NSs (see Section 6.1). This is a plausible hypothesis and we discuss this in the next section.

\footnotetext{
13 The radar albedo of asteroids ranges from 0.05 for carbonaceous chondrites to 0.6 for high-density nickel-iron. Typical value is 0.15 .
}

To conclude, the analysis of Bower et al. (2007) and our own analysis do not favor association of the long-duration radio transients with many types of astrophysical sources. However, we cannot rule out association of the long-duration radio transients with Galactic isolated NSs, Galactic cool brown dwarfs, or some sort of exotic explosion. Although cool brown dwarfs are interesting candidates, they require an emission mechanism which does not produce circularly polarized radiation like that observed in flare stars.

\section{ISOLATED OLD NEUTRON STARS AS PROGENITORS OF THE LONG-DURATION RADIO TRANSIENTS}

The organization of this section is as follows. In Section 6.1, we give a brief introduction to old NSs. Based on Monte Carlo orbital simulations of NSs, we estimate their sky surface density, distances, and source number counts function (Section 6.2). In Section 6.3, we discuss the energetics of long-duration radio transients in the context of Galactic NS. In Section 6.4, we present a simple synchrotron emission model for such radio flares, while in Section 6.5 we discuss the possibility that these are some kind of intermittent pulsars. Finally, we derive lower and upper limits on the distance scale to the Kida et al. transients assuming they are originating from Galactic NSs (Section 6.6).

\subsection{Introduction to Galactic Isolated Old NS}

Based on the metal content of the Milky Way galaxy, Arnett et al. (1989) estimated that about $10^{9} \mathrm{SNe}$ exploded in the Milky Way and hence similar number of NSs were born in our Galaxy. The observed SN rate in the Galaxy suggests a number which is smaller by a factor of 3-10. Thus, it is expected that there are about $10^{8}$ to $10^{9} \mathrm{NSs}$ in the Galaxy.

NSs cool on relatively short timescales $\left(\sim 10^{6} \mathrm{yr}\right.$; e.g., Yakovlev \& Pethick 2004). Therefore, they are expected to be intrinsically dim and extremely hard to detect. However, Ostriker et al. (1970) suggested that NSs may be heated through accretion of interstellar matter (ISM). This novel suggestion raised many hopes that NSs may be detected as soft X-ray sources (e.g., Helfand et al. 1980; Treves \& Colpi 1991; Blaes \& Madau 1993). These predictions were followed by intensive searches for such objects (e.g., Motch et al. 1997; Maoz et al. 1997; Haberl et al. 1998; Rutledge et al. 2003; Agüeros et al. 2006). However, the several candidates that were found (e.g., Haberl et al. 1998) are young cooling NSs (e.g., Neuhäuser \& Trümper 1999; Popov et al. 2000; Treves et al. 2001).

Among the usual explanations for the observational paucity of isolated accreting old NSs in the ROSAT all-sky survey (Voges et al. 1999) is the fact that the velocity distribution of NSs is much higher than the early estimates (e.g., Narayan \& Ostriker 1990 versus Cordes \& Chernoff 1998) or the suggestion that magnetized NSs cannot accrete matter efficiently at the Bondi-Hoyle rate ${ }^{14}$ (e.g., Colpi et al. 1998; Perna et al. 2003).

\subsection{Surface Density and Distance}

In order to estimate the surface density, we need a model for isolated old NS space distribution at the current epoch. Ofek (2009) integrated the orbits of simulated NSs in the Galactic gravitational potential, using two different natal velocity distributions and vertical scale height distributions of the

\footnotetext{
14 Bondi \& Hoyle (1944).
} 


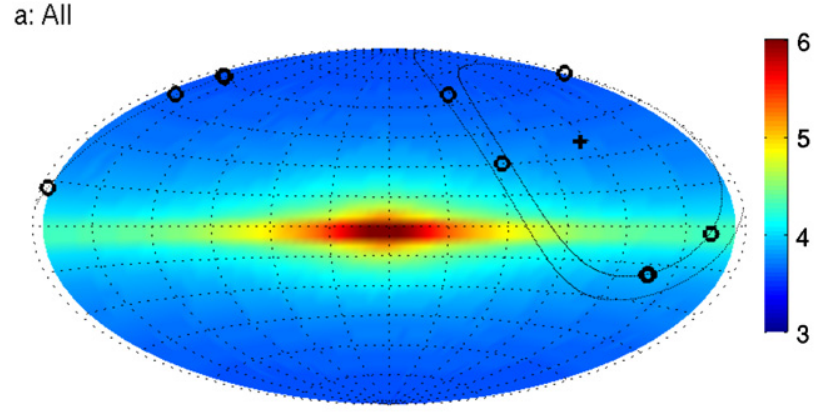

b: $\mathrm{d}<1 \mathrm{kpc}$

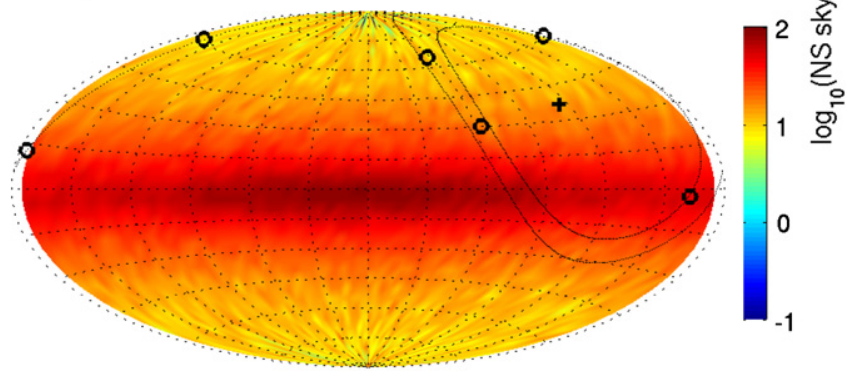

Figure 6. Sky surface density distribution of all NSs (panel (a)) and NSs at distance $<1 \mathrm{kpc}$ from the Sun (panel (b)). The maps are presented in the Galactic coordinate system and using the Aitoff equal area projection. The sky surface densities are calculated using the catalog of simulated old NSs presented in Ofek (2009) including both populations of bulge-born and disk-born NSs, and using the natal velocity distribution of Arzoumanian et al. (2002) and assuming $10^{9} \mathrm{NSs}$. The plus sign marks the position of the Bower et al. (2007) field at $l=115^{\circ}, b=37^{\circ}$. The lines mark the declination zone of $32^{\circ}-42^{\circ}$, in which the survey for radio transients described by Kida et al. (2008) was conducted. The 11 circles mark the position of the bright (flux at $1.4 \mathrm{GHz}$ greater than $1 \mathrm{Jy}$ ) transients reported by Kida et al. (2008).

progenitor populations suggested by Arzoumanian et al. (2002) and Faucher-Giguère \& Kaspi (2006). These simulations assume that $60 \%$ of the Galactic NSs were born in the Galactic bulge 12 Gyr ago, while $40 \%$ were born continuously, with a constant rate, in the disk over the past $12 \mathrm{Gyr}$.

Based on this catalog, and using the Arzoumanian et al. initial conditions, in Figure 6 we show the theoretical sky surface density of all Galactic NSs, and NSs within $1 \mathrm{kpc}$ from the Sun. Toward the direction of the Bower et al. (2007) field, the total surface density of isolated old NSs is about $10^{4} \mathrm{deg}^{-2}$. The mean surface density of old NSs at Galactic latitude $b>30^{\circ}$, which roughly corresponds to the FIRST survey footprint, is about 5\% larger. The small difference between the two surface densities suggests that if indeed long-duration radio transients are associated with isolated old NSs then the comparison between the $1.4 \mathrm{GHz}$ and $5 \mathrm{GHz}$ rates presented in Section 4.3 is not affected by the different sky positions at which these surveys were conducted.

In Figure 7, we show the cumulative surface density of NSs, at the Bower et al. (2007) field direction, as a function of distance from the observer (i.e., the Sun). The plots assume that the Sun is located $8 \mathrm{kpc}$ from the Galactic center (Ghez et al. 2008). In Figure 7, we also mark, by a dotted horizontal line, the minimal sky surface density of long-duration radio transients (i.e., $\Sigma>60 \mathrm{deg}^{-2}$ ) that we derived in Section 4.2. This figure suggests that if isolated old NSs are indeed related to the sub-mJy level Bower et al. transients then their typical distance scale is at least $\sim 1 \mathrm{kpc}$. Otherwise the predicted sky surface density will not be consistent with the minimum sky

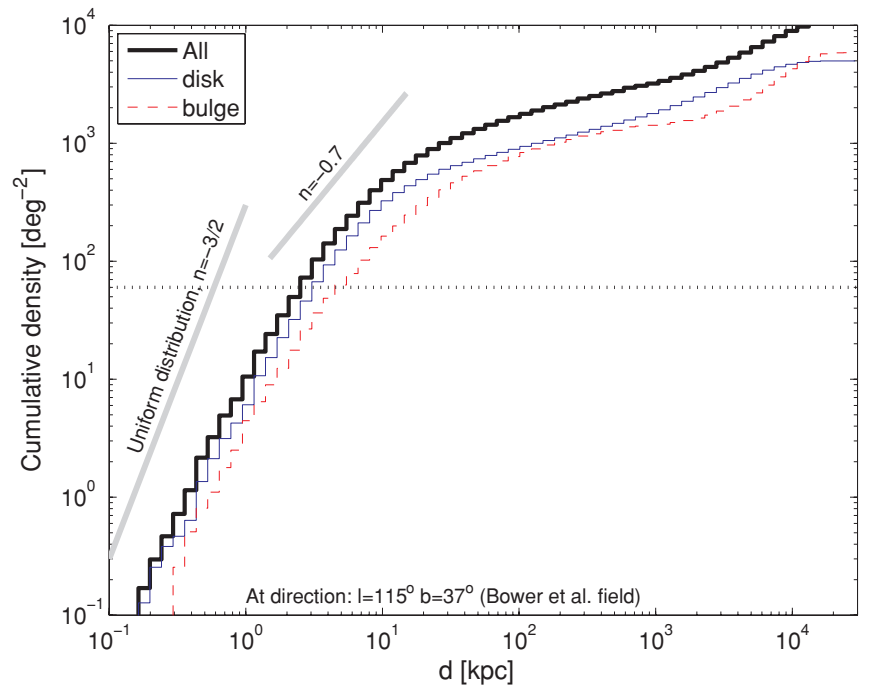

Figure 7. Cumulative number of simulated NSs per $\mathrm{deg}^{2}$ at the direction of the Bower et al. (2007) field as a function of distance. The thick solid line shows the distribution of the entire NS population of which $60 \%(40 \%)$ are assumed to be of bulge (disk) origin, the thin solid line is for the disk-born NSs, while the dashed line is for the bulge-born NSs. The three distributions give a rough idea regarding the uncertainties that may arise from our ignorance regarding the birth locations of NSs along Galactic history. The dotted horizontal line marks the minimum surface density required $\left(60 \mathrm{deg}^{-2}\right.$; see Section 4.2$)$. The thick solid gray lines show the expected slopes for a population with a homogeneous distribution $(n=-3 / 2)$, and $n=-0.7$, which corresponds to our $3 \sigma$ upper limit (see however the text).

surface density of NS at the direction of the Bower et al. field (see Section 4.2). On the other hand, the typical distance is presumably not greater than about $5 \mathrm{kpc}$, otherwise the slope of the cumulative distribution will be too shallow relative to the steep power-law index, $n$, of the number count distribution derived in Section 4.3. We note that the luminosity function of these hypothetical events may depend on the age (and therefore distance) to the NSs. Therefore, we do not attempt to quantify the upper limit on the distance mentioned above.

A possibility that we should consider is that only a fraction of the Galactic NSs are the progenitors of the long-duration radio transients (e.g., only "young" NSs). Based on the NSs orbital simulations, we find that all the NSs with ages smaller than at least $1 \mathrm{Gyr}$ are required as progenitors of the radio transients. Thus, the sub-mJy long-duration radio transients cannot arise from pulsars.

A simple test for the hypothesis that long-duration radio transients are associated with Galactic isolated old NSs (or for that matter any Galactic population with scale height of a kpc or so) is to look for excess of sub-mJy radio transients near the Galactic center, relative to high Galactic latitude. In Figure 8, we therefore show the cumulative distribution of isolated old NSs as a function of distance, but toward the direction of the Galactic center.

There are several marked differences between Figures 7 and 8. The total sky surface density in the direction of the Galactic center is about 2 orders of magnitude larger than the sky surface density at the direction of the Bower et al. field. Next, if we consider only NSs with distances up to $1 \mathrm{kpc}$, then the sky surface density in the direction of the Galactic center is about seven times larger than that in the Bower et al. field. Finally, the source count function in the direction of the Galactic center is steeper than that in the direction of the Bower et al. (2007) field. 


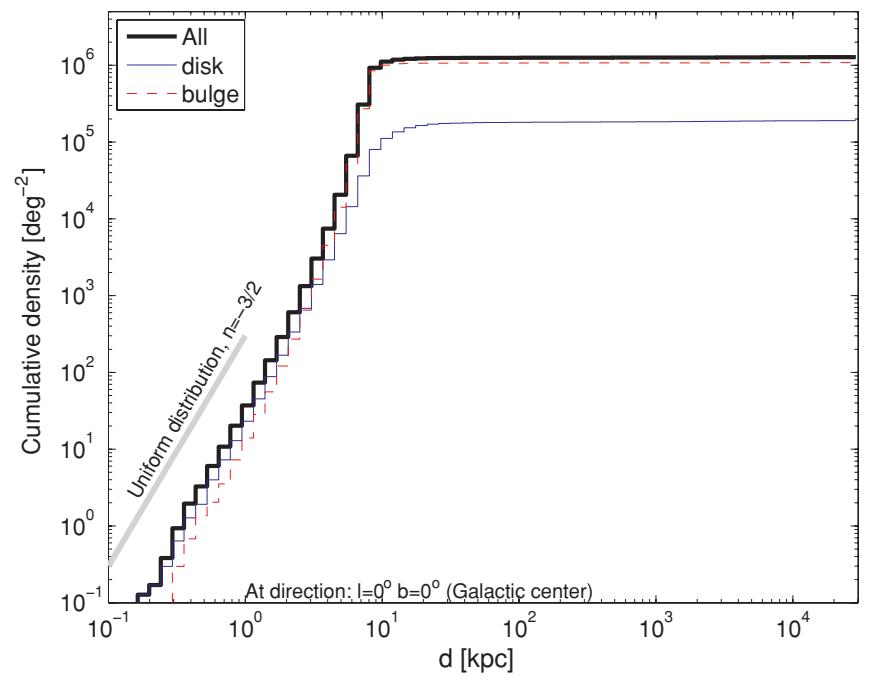

Figure 8. Same as Figure 7, but toward the direction of the Galactic center. (A color version of this figure is available in the online journal.)

\subsection{Energetics and Timescales}

In the old-NS framework the typical energy release from a single flare is

$$
\begin{aligned}
E_{\mathrm{f}} \approx & 2 \times 10^{32} \frac{S_{v}}{0.4 \mathrm{mJy}}\left(\frac{d}{1 \mathrm{kpc}}\right)^{2} \\
& \times \frac{\Delta v}{10^{10} \mathrm{~Hz}} \frac{t_{\text {dur }}}{0.5 \text { day }} \mathrm{erg} .
\end{aligned}
$$

The rate of the sub-mJy long-duration radio transients is in the range of $\Re \approx 20 \mathrm{deg}^{-2} \mathrm{yr}^{-1}$ to $2 \times 10^{4} \mathrm{deg}^{-2} \mathrm{yr}^{-1}$ (Equation (1)). Adopting a representative rate of $\Re \approx 500 \mathrm{deg}^{-2} \mathrm{yr}^{-1}$, and $t_{\text {dur }} \sim 0.5$ day we find that over the Hubble time the allsky number of events is larger by several orders of magnitude than that of any known Galactic stellar population. Therefore, any hypothesis involving Galactic stars would require that the sources be repeaters. The mean timescale between bursts, $\Delta t_{b}$, is given by

$$
\begin{aligned}
\Delta t_{b} & =\frac{\Sigma}{\Re} \\
& \cong 0.2 \frac{\Sigma}{10^{2} \mathrm{deg}^{-2}}\left(\frac{\Re}{500}\right)^{-1} \mathrm{yr} \\
& \cong 0.2 \frac{\Sigma}{10^{2} \mathrm{deg}^{-2}} \frac{t_{\text {dur }}}{0.5 \text { day }} \mathrm{yr},
\end{aligned}
$$

where $\Sigma$ is the sky surface density of sources in the direction of the Bower et al. (2007) field. In that case, the flares' duty cycle is of order of $\mathcal{D}=t_{\mathrm{dur}} / \Delta t_{b} \approx 7 \times 10^{-3}$. An immediate prediction is that if long-duration radio transients are associated with Galactic NSs, then the repetition timescale for bursts is relatively short, of the order of several months.

Multiplying Equation (10) by the expected number of flares within the Hubble time $\left(t_{\mathrm{H}} / \Delta t_{b} \sim 5 \times 10^{10}\right.$, where $t_{\mathrm{H}}$ is the Hubble time), we find that the total energy emitted by a single object over its lifetime is

$$
\begin{aligned}
E_{\mathrm{tot}} \approx & 1 \times 10^{43} \frac{S_{v}}{0.4 \mathrm{mJy}}\left(\frac{d}{1 \mathrm{kpc}}\right)^{2} \frac{\Delta v}{10^{10} \mathrm{~Hz}} \\
& \times\left(\frac{\Sigma}{10^{2} \mathrm{deg}^{-2}}\right)^{-1} \mathrm{erg} .
\end{aligned}
$$

Dividing Equation (10) by Equation (11), the mean luminosity over time required in order to power these bursts is

$$
\begin{aligned}
\langle\dot{E}\rangle \approx 3 & \times 10^{25} \frac{S_{v}}{0.4 \mathrm{mJy}}\left(\frac{d}{1 \mathrm{kpc}}\right)^{2} \frac{\Delta v}{10^{10} \mathrm{~Hz}} \\
& \times\left(\frac{\Sigma}{10^{2} \mathrm{deg}^{-2}}\right)^{-1} \mathrm{erg} \mathrm{s}^{-1} .
\end{aligned}
$$

Note that this quantity is independent of $\Delta t_{b}$.

Next, we consider the energy reservoir of isolated old NSs, and check whether it is consistent with the mean luminosity given by Equation (13). Isolated old NSs have several sources of available energy. Among these are (1) spin energy, (2) magnetic energy, and (3) accretion, from the ISM, energy.

The rotational kinetic energy of NSs is

$$
\begin{aligned}
E_{\mathrm{rot}} & =\frac{1}{2} I \omega^{2} \\
& \cong 2.2 \times 10^{44}\left(\frac{P}{10 \mathrm{~s}}\right)^{-2} \frac{M_{\mathrm{NS}}}{1.4 M_{\odot}}\left(\frac{R_{\mathrm{NS}}}{10 \mathrm{~km}}\right)^{2} \mathrm{erg}
\end{aligned}
$$

where $M_{\mathrm{NS}}$ is the NS mass, $I$ is the moment of inertia (assuming $\left.I=0.4 M_{\mathrm{NS}} R_{\mathrm{NS}}^{2}\right), \omega$ is the NS rotational angular speed, and $P$ $(=2 \pi / \omega)$ is its rotation period. The energy-loss rate available from the rotational energy reservoir is

$$
\begin{aligned}
\dot{E}_{\mathrm{rot}}= & I \omega \dot{\omega}=4 \pi^{2} I P^{-3} \dot{P} \\
\cong & 4.4 \times 10^{26} \frac{M_{\mathrm{NS}}}{1.4 M_{\odot}}\left(\frac{R_{\mathrm{NS}}}{10 \mathrm{~km}}\right)^{2} \\
& \times\left(\frac{P}{10 \mathrm{~s}}\right)^{-3} \frac{\dot{P}}{10^{-17}} \mathrm{erg} \mathrm{s}^{-1},
\end{aligned}
$$

where $\dot{\omega}$ is the time derivative of the NS angular frequency and $\dot{P}$ is defined as minus of its period derivative. Typical values for $\dot{P}$ are in the range of $10^{-10} \mathrm{~s} \dot{\mathrm{s}}$ for magnetars, $10^{-17} \mathrm{~s} \dot{\mathrm{s}}$ to $10^{-12} \mathrm{~s} \dot{\mathrm{s}}$ for normal radio pulsars, and $10^{-21} \mathrm{~s} \dot{\mathrm{s}}$ to $10^{-18} \mathrm{~s} \dot{\mathrm{s}}$ for millisecond pulsars (Srinivasan 1989).

A large fraction of NSs may have high surface magnetic field in excess of $10^{14} \mathrm{G}$ (e.g., Magnetars). The energy stored in the magnetic field of such objects is about

$$
\begin{aligned}
E_{B} & \approx \frac{4}{3} \pi R_{\mathrm{NS}}^{3} \frac{B^{2}}{8 \pi} \\
& \approx 1.7 \times 10^{45}\left(\frac{r_{\mathrm{NS}}}{10 \mathrm{~km}}\right)^{3}\left(\frac{B}{10^{14} \mathrm{G}}\right)^{2} \mathrm{erg},
\end{aligned}
$$

where $R_{\mathrm{NS}}$ is the NS radius and $B$ is its interior mean magnetic field. We note that the internal magnetic field may be higher, and therefore, Equation (16) is a lower limit on the magnetic energy reservoir. The rate at which the magnetic field of NSs is decaying is debated both theoretically and observationally (e.g., Urpin \& Muslimov 1992; Chanmugam 1992; Phinney \& Kulkarni 1994; Sengupta 1997; Sun \& Han 2002). Assuming the magnetic field is uniformly decaying on the Hubble timescale, this will provide, on average, energy-loss rate of $\dot{E} \approx 4 \times 10^{27} \mathrm{erg} \mathrm{s}^{-1}$.

Another possible source of energy is heating of the NS by accretion from the ISM (e.g., Ostriker et al. 1970). Assuming NSs accrete at the Bondi-Hoyle rate (Bondi \& Hoyle 1944), the energy-loss rate will be 


$$
\begin{aligned}
\dot{E}_{\mathrm{acc}}= & \frac{4 \pi G^{3} M_{\mathrm{NS}}^{3} \rho_{\mathrm{ISM}}}{R_{\mathrm{NS}}\left(v^{2}+c_{s}^{2}\right)^{3 / 2}} \\
\approx & 5 \times 10^{26}\left(\frac{M_{\mathrm{NS}}}{1.4 M_{\odot}}\right)^{3}\left(\frac{R_{\mathrm{NS}}}{10 \mathrm{~km}}\right)^{-1} \\
& \times \frac{n_{\mathrm{ISM}}}{0.1 \mathrm{~cm}^{-3}}\left(\frac{v}{300 \mathrm{~km} \mathrm{~s}^{-1}}\right)^{-3} \mathrm{erg} \mathrm{s}^{-1},
\end{aligned}
$$

where $v$ is the velocity of the NS relative to an ISM with a mass (number) density $\rho_{\mathrm{ISM}}\left(n_{\mathrm{ISM}}\right) . c_{s}$ is the sound speed in the ISM, which is of the order of $10 \mathrm{~km} \mathrm{~s}^{-1}$ and is therefore neglected. ${ }^{15}$ We note, however, that magneto hydrodynamic simulations suggest that in the presence of a strong magnetic field the accretion rate will be suppressed relative to the Bondi rate (e.g., Toropina et al. 2001, 2003, 2005; see, however, Arons $\&$ Lea 1976, 1980).

To conclude, the combination of NSs energy sources may provide enough energy to explain long-duration radio transients. Specifically, the rotation energy of NSs and the energy available for NSs from accretion from the ISM are at least an order of magnitude larger than needed for generating the long-duration radio transients (Equation (13)). However, unless additional energy sources are invoked, this implies that the outbursts cannot emit much higher energy at other frequencies.

\subsection{Incoherent Synchrotron Radiation from an Afterglow}

Now we address the mechanism of radio emission. There are two possibilities: (1) incoherent synchrotron emission (the afterglow model) and (2) coherent emission. We discuss the first possibility in this section and the second possibility in Section 6.5.

In the afterglow model, the source undergoes an explosive event and ejects relativistic particles and may generate magnetic field (hereafter relativistic plasma). Some sort of pressure confinement is needed to prevent rapid expansion of the relativistic plasma. Otherwise the expansion or adiabatic losses vastly increase the energy budget which would be inconsistent with the old NS framework. In the case of GRBs, the afterglow is confined by the dynamic pressure of the blast wave. We return to this critical issue toward the end of the subsection. We proceed by computing the (quasi)static properties of the (approximately) confined relativistic plasma. We note that the parameters derived from this model are estimated to within an order of magnitude.

Our simple model involves four free parameters: the radius of the emitting region, $R$; the mean electron density, $n_{e}$; the magnetic field, $B$; and the characteristic electron Lorentz factor, $\gamma_{e}=\left(1-\beta_{e}^{2}\right)^{-1 / 2}$, where $\beta_{e}$ is the typical electron speed in units of the speed of light. The large value of the $5 \mathrm{GHz}$ rate (Equation (1)) relative to that at $1.4 \mathrm{GHz}$ rate (Equation (2)) suggests that the synchrotron self-absorption frequency is above $5 \mathrm{GHz}$ (see Section 4.3). Therefore, we assume $v_{s} \gtrsim 5 \mathrm{GHz}$, and that the optical depth at $5 \mathrm{GHz}, \tau_{5}>1$. Furthermore, we assume that the optical depth at the synchrotron frequency $\tau_{s} \lesssim 1$.

\footnotetext{
15 The sound speed is given by $c_{s}=\left(\gamma k_{B} T / \mu_{m}\right)^{1 / 2}$, where $\gamma$ is the adiabatic index, $k_{B}$ is the Boltzmann constant, $T$ is the temperature, and $\mu_{m}$ is the mean weight of the ISM particles. For $\gamma=5 / 3$ and $T=10^{4} \mathrm{~K}$, the sound speed is 11 and $15 \mathrm{~km} \mathrm{~s}^{-1}$ for neutral and ionized gas, respectively.
}

In order to estimate these parameters, we use the following relations:

1. The characteristic synchrotron frequency:

$$
\begin{aligned}
v_{s} & =\gamma_{e}^{2} \frac{e B}{2 \pi m_{e} c} \\
& \cong 2.8 \times 10^{6} \gamma_{e}^{2} B \mathrm{~Hz},
\end{aligned}
$$

where $e$ is the elementary (electron) charge, $m_{e}$ is the electron mass, and $B$ the magnetic field in cgs units (i.e., Gauss).

2. The power emitted by a relativistic single electron due to synchrotron radiation (e.g., Rybicki \& Lightman 1979) is

$$
\begin{aligned}
P_{s} & =\frac{4}{3} \sigma_{T} c \beta_{e}^{2} \gamma_{e}^{2} U_{B} \\
& \cong 1.1 \times 10^{-15} \beta_{e}^{2} \gamma_{e}^{2} B^{2} \mathrm{erg} \mathrm{s}^{-1},
\end{aligned}
$$

where $\sigma_{T}$ is the Thomson cross section and $U_{B}=B^{2} /(8 \pi)$ is the magnetic field energy density. The synchrotron cooling timescale, assuming $\tau_{s} \lesssim 1$, is given by

$$
\begin{aligned}
t_{\mathrm{syn}} & =\frac{\gamma_{e} m_{e} c^{2}}{P_{s}} \\
& \cong 7.7 \times 10^{8} \gamma_{e}^{-1} \beta_{e}^{-2} B^{-2} \mathrm{~s} .
\end{aligned}
$$

3. The brightness temperature, $T_{B}$ (essentially a conveniently chosen surrogate for distance) is

$$
\begin{aligned}
T_{B}= & S_{\nu} d^{2}(c / \nu)^{2}\left(2 \pi k_{B} R^{2}\right)^{-1} \\
\cong & 1.6 \times 10^{21} \frac{S_{\nu}}{0.4 \mathrm{mJy}}\left(\frac{d}{1 \mathrm{kpc}}\right)^{2} \\
& \times\left(\frac{v}{5 \mathrm{GHz}}\right)^{-2}\left(\frac{R}{10 \mathrm{~km}}\right)^{-2} \mathrm{~K},
\end{aligned}
$$

where $v$ is the frequency at which we observe (i.e., $5 \mathrm{GHz}$ ). For optical depth larger than unity the brightness temperature is related to the electrons energy:

$$
k_{B} T_{B} \approx \gamma_{e} m_{e} c^{2} .
$$

4. Finally, in order to get a self-absorption spectrum (see Section 4.3) the optical depth at $5 \mathrm{GHz}$ should be larger than unity. This holds if and only if at $5 \mathrm{GHz}$ the thermal emission is smaller than the optically thin emission:

$$
2 \pi k_{B} T_{B}\left(\frac{v}{c}\right)^{2}<\frac{1}{3} R n_{e} \frac{P_{s}}{v_{s}}\left(\frac{v}{v_{s}}\right)^{1 / 3} .
$$

Next, we can solve Equations (18)-(23) for the free parameters $B, \gamma_{e}$, and $R$ and we can put a lower limit on the value of $n_{e}$. Since we do not know the exact value of $t_{\mathrm{dur}}, v_{s}$, and $d$ we solve for the free parameters as a function of these arguments. For completeness, we also state the dependency on $S_{\nu}$. We normalized the solutions for $S_{v}=0.4 \mathrm{mJy}, d=1 \mathrm{kpc}$, $t_{\text {syn }} \approx t_{\text {dur }}=0.5$ day, and $v_{s}=2 \times 10^{11} \mathrm{~Hz}$ (the choice for $v_{s}$ is to minimize the total energy; see below). We note that $t_{\mathrm{dur}} \approx t_{\mathrm{syn}}$ means that there is no energy injection into the emission region after the initial burst (i.e., the duration of the events is dominated by the synchrotron cooling timescale). However, if energy is injected then $t_{\mathrm{dur}} \gtrsim t_{\mathrm{syn}}$. 
The following solution holds for $\tau_{5} \gtrsim 1$ and $\tau_{s} \lesssim 1$ :

$$
\begin{aligned}
& B \approx 17\left(\frac{v_{s}}{2 \times 10^{11} \mathrm{~Hz}}\right)^{-1 / 3}\left(\frac{t_{\mathrm{syn}}}{0.5 \text { day }}\right)^{-2 / 3} \mathrm{G} \\
& \gamma_{e} \approx 66\left(\frac{v_{s}}{2 \times 10^{11} \mathrm{~Hz}}\right)^{2 / 3}\left(\frac{t_{\mathrm{syn}}}{0.5 \text { day }}\right)^{1 / 3}, \\
& R \approx 6 \times 10^{10}\left(\frac{v_{s}}{2 \times 10^{11} \mathrm{~Hz}}\right)^{-1 / 3}\left(\frac{t_{\mathrm{syn}}}{0.5 \text { day }}\right)^{-1 / 6} \\
& \quad \times\left(\frac{S_{v}}{0.4 \mathrm{mJy}}\right)^{1 / 2}\left(\frac{d}{1 \mathrm{kpc}}\right) \mathrm{cm},
\end{aligned}
$$

and the lower limit on $R n_{e}$ is

$$
R n_{e}>2 \times 10^{16}\left(\frac{v_{s}}{2 \times 10^{11} \mathrm{~Hz}}\right)^{4 / 3} \frac{t_{\mathrm{syn}}}{0.5 \mathrm{day}} \mathrm{cm}^{-2}
$$

We note that in order to minimize the energy, $n_{e}$ should be around the lower limit implied by Equation (27). For convenience, we also give the brightness temperature:

$$
\begin{aligned}
T_{B} \approx & \times 10^{11}\left(\frac{v_{s}}{2 \times 10^{11} \mathrm{~Hz}}\right)^{2 / 3} \\
& \times\left(\frac{t_{\text {syn }}}{0.5 \text { day }}\right)^{1 / 3} \mathrm{~K} .
\end{aligned}
$$

Next, we can derive the ratio between the magnetic energy and electron energy densities:

$$
\begin{aligned}
U_{B} / U_{e}= & \frac{B^{2} /(8 \pi)}{n_{e} \gamma_{e} m_{e} c^{2}} \\
\lesssim & 1\left(\frac{v_{s}}{2 \times 10^{11} \mathrm{~Hz}}\right)^{-3}\left(\frac{t_{\text {syn }}}{0.5 \text { day }}\right)^{-17 / 6} \\
& \times\left(\frac{S_{v}}{0.4 \mathrm{mJy}}\right)^{1 / 2}\left(\frac{d}{1 \mathrm{kpc}}\right),
\end{aligned}
$$

where $U_{e}=n_{e} \gamma_{e} m_{e} c^{2}$ is the electrons' energy density. We note that $U_{B} / U_{e}$ is very sensitive to both the duration, $t_{\mathrm{syn}}$, and the synchrotron frequency, $v_{s}$, whose values are not well known. Therefore, a small change in these unknown parameters will change $U_{B} / U_{e}$ dramatically (see also Readhead 1994).

We note that by setting $U_{B} / U_{e} \sim 1$, we have ensured that $T_{B}$ is equal to the equipartition brightness temperature (Readhead 1994) and minimized the energy requirement. Next, we test if these parameters are below the inverse Compton catastrophe limit (Kellermann \& Pauliny-Toth 1969). The inverse Compton catastrophe is relevant if $U_{\mathrm{rad}} / U_{B} \gtrsim 1$. Assuming $\tau_{s} \lesssim 1$

$$
U_{\mathrm{rad}} \approx \frac{R U_{e}}{c t_{\mathrm{syn}}}
$$

Since $R /\left(c t_{\text {syn }}\right) \ll 1$ we get $U_{\text {rad }} \ll U_{e} \sim U_{B}$. Therefore, inverse Compton effects can be neglected.

Assuming the electron density is close to the minimum density implied by Equation (27), the total energy in a single

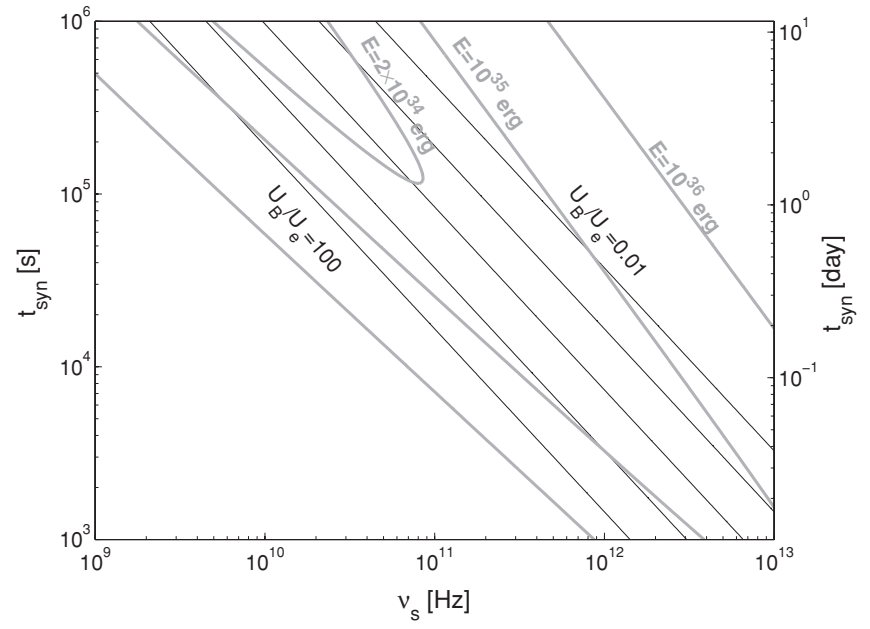

Figure 9. $U_{B} / U_{e}$ (black contours) and total energy (gray contours), $E=$ $4 / 3 \pi R^{3}\left(U_{e}+U_{B}\right)$, as a function of $v_{s}$ and $t_{\mathrm{syn}}$, and assuming $d=1 \mathrm{kpc}$, and $S_{v}=0.4 \mathrm{mJy}$. The equal $U_{B} / U_{e}$ lines are $100,10,1,0.1$, and 0.01 from left to right.

burst (i.e., in both the electrons and magnetic field) is given by

$$
\begin{aligned}
E= & \frac{4}{3} \pi R^{3}\left(U_{e}+U_{B}\right) \\
\cong & 1 \times 10^{34}\left[\left(\frac{v_{s}}{2 \times 10^{11} \mathrm{~Hz}}\right)^{4 / 3}\left(\frac{t_{\mathrm{syn}}}{0.5 \mathrm{day}}\right)\right. \\
& \left.\times\left(\frac{S_{v}}{0.4 \mathrm{mJy}}\right)\left(\frac{d}{1 \mathrm{kpc}}\right)^{2}\right] \\
& +1 \times 10^{34}\left[\left(\frac{v_{s}}{2 \times 10^{11} \mathrm{~Hz}}\right)^{-5 / 3}\left(\frac{t_{\mathrm{syn}}}{0.5 \mathrm{day}}\right)^{-11 / 6}\right. \\
& \left.\times\left(\frac{S_{v}}{0.4 \mathrm{mJy}}\right)^{3 / 2}\left(\frac{d}{1 \mathrm{kpc}}\right)^{3}\right] \mathrm{erg} .
\end{aligned}
$$

The specific values we have selected, $v_{s}=2 \times 10^{11} \mathrm{~Hz}$ and $t_{\text {syn }}=0.5$ day, give a solution which is near equipartition and therefore minimizes the energy. For $n_{e}$ close to its minimum implied by Equation (27), the total energy in a burst, and $U_{B} / U_{e}$ as a function of $v_{s}$ and $t_{\text {syn }}$ are shown in Figure 9. This figure suggests that the minimum energy required per burst is around $10^{34} \mathrm{erg}$. This energy per burst multiplied by the expected number of bursts over the Hubble time $\left(\sim 5 \times 10^{10}\right)$ is of the same order of magnitude of the energy reservoir of NSs identified in Section 6.3.

So far we have assumed that the duration of the events is set by the synchrotron cooling timescale. We now explore the consequences of decreasing the cooling timescale and letting the duration be determined by the plasma injection timescale. In this case, Equation (31) represents the total energy of a flare within the synchrotron cooling timescale. Therefore, in order to get the total energy we need to multiply Equation (31) by $t_{\mathrm{dur}} / t_{\mathrm{syn}}$. As can be gathered from Figure 9, the energy is minimized by setting the cooling time equal to the duration time.

It is interesting to compare the derived radius, $R \approx 6 \times$ $10^{10} \mathrm{~cm}$, with some typical radii dominating NS physical processes. The light-cylinder radius is

$$
R_{\mathrm{lc}}=\frac{c}{2 \pi} P \cong 4.8 \times 10^{10} \frac{P}{10 \mathrm{~s}} \mathrm{~cm} .
$$


The co-rotation radius of a NS is

$$
\begin{aligned}
R_{\text {cor }} & =\left(\frac{G M_{\mathrm{NS}}}{\omega^{2}}\right)^{1 / 3} \\
& \cong 7.8 \times 10^{8}\left(\frac{M_{\mathrm{NS}}}{1.4 M_{\odot}}\right)^{1 / 3}\left(\frac{P}{10 \mathrm{~s}}\right)^{2 / 3} \mathrm{~cm} .
\end{aligned}
$$

Assuming a Bondi-Hoyle accretion rate,

$$
\dot{M}=\frac{4 \pi G^{2} M_{\mathrm{NS}}^{2} m_{p} n_{\mathrm{ISM}}}{\left(V^{2}+c_{s}^{2}\right)^{3 / 2}},
$$

where $m_{p}$ is the proton mass, then the magnetosphere radius is

$$
\begin{aligned}
R_{m}= & \left(\frac{\mu^{2}}{\dot{M} \sqrt{2 G M_{\mathrm{NS}}}}\right)^{2 / 7} \\
\cong & 2.1 \times 10^{11}\left(\frac{\mu}{4 \times 10^{30}}\right)^{4 / 7}\left(\frac{\dot{M}}{2 \times 10^{8} \mathrm{gr} \mathrm{s}^{-1}}\right)^{-2 / 7} \\
& \times\left(\frac{M_{\mathrm{NS}}}{1.4 M_{\odot}}\right)^{-1 / 7} \mathrm{~cm},
\end{aligned}
$$

where $\mu$ is the magnetic dipole moment of the NS. Finally, the accretion radius is

$$
\begin{aligned}
R_{\mathrm{acc}} & \approx \frac{2 G M_{\mathrm{NS}}}{V^{2}} \\
& \approx 1.7 \times 10^{12} \frac{M_{\mathrm{NS}}}{1.4 M_{\odot}}\left(\frac{V}{150 \mathrm{~km} \mathrm{~s}^{-1}}\right)^{-2} \mathrm{~cm} .
\end{aligned}
$$

As noted at the beginning of the subsection rapid expansion of the radiating plasma would vastly increase the energy budget. For this reason, an integral requirement of the incoherent model is that the plasma must be confined (in which case the duration of the event is set by the cooling time or by the duration of the injection of energy by the source). We note that the confinement radius should probably be smaller than the lightcylinder radius. Otherwise, the energy requirement will be larger due to the inertia of the electrons. The equipartition radius we find (Equation (26)) is a little bit larger than the plausible confinement radius (e.g., Equation (32)). However, our calculation provides only an order of a magnitude estimate to the fireball parameters and the equipartition radius in particular. Therefore, we cannot rule out the incoherent synchrotron model based on the small inconsistency between the equipartition radius and light-cylinder radius.

Assuming a dipole magnetic field, decaying as $R^{-3}$ (inside the light-cylinder radius; Equation (32)), and $B=17 \mathrm{G}$ at $R=6 \times 10^{10} \mathrm{~cm}$, the extrapolated magnetic field strength on the surface of a $10 \mathrm{~km}$ radius NS will be about

$$
\begin{aligned}
B(10 \mathrm{~km}) \approx & B\left(\frac{R}{10 \mathrm{~km}}\right)^{3} \\
\approx & 4 \times 10^{15}\left(\frac{v_{s}}{2 \times 10^{11} \mathrm{~Hz}}\right)^{-4 / 3}\left(\frac{t_{\mathrm{syn}}}{0.5 \text { day }}\right)^{-7 / 6} \\
& \times\left(\frac{S_{v}}{0.4 \mathrm{mJy}}\right)^{3 / 2}\left(\frac{d}{1 \mathrm{kpc}}\right)^{3} \mathrm{G} .
\end{aligned}
$$

This is higher than the typical estimated surface magnetic fields of pulsars and magnetars. However, for somewhat larger $v_{s}$ or $t_{\text {syn }}$ the discrepancy is smaller. Moreover, the events may be related to the release of magnetic energy stored in the NS interior. Therefore, we conclude that the incoherent synchrotron model cannot be ruled out.

Finally, we note that the total mass of matter within the emission radius is $4 / 3 \pi n_{e} m_{p} R^{3} \sim 4 \times 10^{14} \mathrm{gr}$. Interestingly, this mass is similar to the total amount of matter that a NS with a space velocity of $150 \mathrm{~km} \mathrm{~s}^{-1}$ will accrete from the ISM (assuming $n=0.1 \mathrm{~cm}^{-3}$ and Bondi-Hoyle accretion) within several months, which is the typical time interval between bursts that we found in Section 6.3. We note that Treves et al. (1993) suggested that accreted matter from the ISM is piled up near the Alfvén radius, followed by infall of the piled up matter on the NS. They estimated that these episodic infalls may occur every several months.

\subsection{Flares from Intermittent Pulsars}

In recent years, several types of pulsars with small duty cycles have been discovered. This includes the RRATs (McLaughlin et al. 2006) and intermittent pulsars (Kramer et al. 2006). Several models were suggested to explain such episodic pulsars (e.g., Treves et al 1993; Zhang et al. 2007; Cordes \& Shannon 2008). However, their evolutionary status is still unclear.

Known intermittent pulsars and RRATs have characteristic ages similar to those of "normal" pulsars (i.e., $\lesssim 10^{7} \mathrm{yr}$ ). However, as we discussed in Section 6.2, the long-duration radio transients cannot be associated exclusively with pulsars younger than about $1 \mathrm{Gyr}$, otherwise their predicted surface density will not be consistent with the observations (Section 4.2).

As shown in Section 6.3, in the framework of Galactic NSs, the duty cycle of the long-duration radio transients is $\sim 7 \times 10^{-3}$. Such a small duty cycle will make it hard to detect them as repeaters in current pulsars searches. In addition, most pulsar searches are conducted at low frequencies $(\lesssim 1.4 \mathrm{GHz})$, in which the rate of the long-duration radio transients seems to be low. Thus, a prediction of this model is that high-frequency (say $5 \mathrm{GHz}$ ) searches should find a much larger rate of long-duration radio transients.

The flat spectrum $(\alpha \gtrsim 0)$ of the long-duration transients is reminiscent of the radio spectrum of magnetars in their "active state" (cf. Camilo et al. 2006). Thus, a plausible model is that the long-duration transients are ancient magnetars in short-lived high states.

\subsection{The Distance Scale to the Kida et al. Transients}

Next, we derive a physical distance to the bright events discussed by Kida et al. (2008). We remind the reader that these events are about a thousand times brighter than the VLA events. We use a modified Rayleigh test (see Fisher et al. 1987) to compare the sky distribution of the Kida et al. (2008) transients with that of the celestial positions of simulated NSs:

$$
\left(\sum_{i=1}^{6} \sin \left[b_{i}\right]\right)^{2} \equiv \Sigma^{2} b,
$$

here $b$ is the Galactic latitude.

Next, we selected from the Heliocentric catalog of simulated isolated old NSs of Ofek (2009), 11 random NSs within the footprints of the Kida et al. search zone, found up to a distance $d$ from the Sun and calculated their $\Sigma^{2} b$. We assume that the survey described in Kida et al. (2008) covers the entire declination zone $\delta=32^{\circ}-42^{\circ}$ equally. For each distance $d$, in the range of $100 \mathrm{pc}$ to $10 \mathrm{kpc}$, we repeated this process 10,000 times. In Figure 10, we show the mean expected value, and the 68, 95, and 99.73 


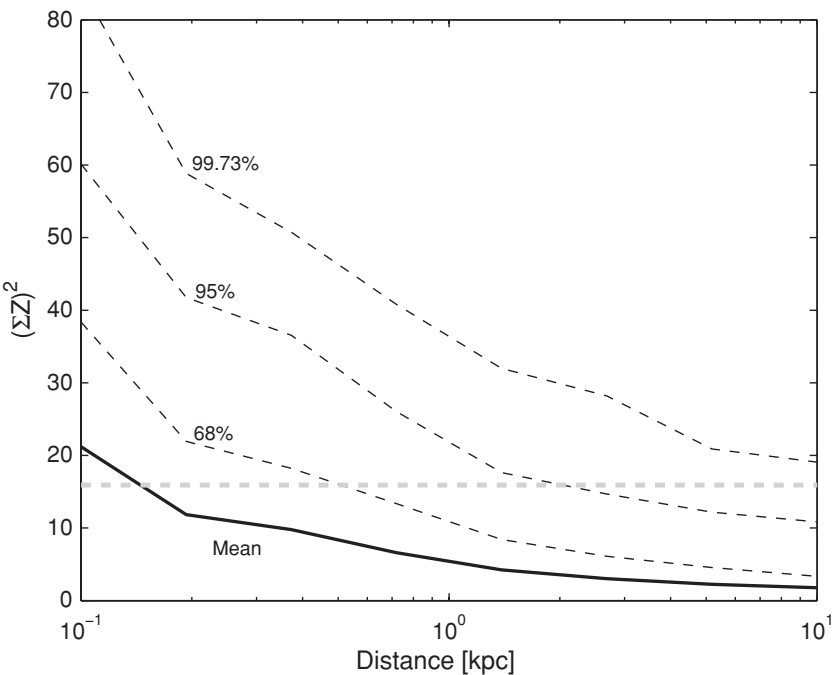

Figure 10. Sky uniformity of the Kida et al. transients (described by $\Sigma^{2} b$; Equation (38)) as a function of the distance to the transients. The gray-thick dashed line shows the value calculated for the observed sample. The black line shows the mean value calculated for the simulated NSs, while the gray-thin dashed lines show the upper 68, 95, and 99.73 percentiles (from bottom up) of the distribution of the uniformity criterion $\Sigma^{2} b$ of the simulated NSs. The lines fluctuate due to the limited statistics.

percentiles, for the distribution of $\Sigma^{2} b$ of simulated NSs as a function of distance. As can be gathered from Figure 10 the typical distance to these events is below $500 \mathrm{pc}(2 \mathrm{kpc})$, at the 68\% (95\%) CL.

Kida et al. reported 11 events in their survey which covers about $7 \%$ of the celestial sphere. Therefore, the all-sky surface density of the Kida et al. transients is $\gtrsim 80$ sources at the $95 \%$ confidence. Ofek (2009) found that the density of NSs in the solar neighborhood is $2 \times 10^{-4} \mathrm{pc}^{-3}$ assuming $10^{9} \mathrm{NS}$ in the Galaxy and the Arzoumanian et al. (2002) initial velocity distribution. At small distances $(\lesssim 100 \mathrm{pc})$ the distribution of NSs around the Sun is near isotropic. Therefore, by comparing the density from the simulations with the observed surface density of the Kida et al. (2008) events, we put a lower limit on the distance to the Kida et al. (2008) event, assuming they originate from Galactic NSs, of $\gtrsim 45$ pc at the $95 \%$ CL.

\section{SUMMARY}

We review several recent discoveries of radio transients with durations between minutes to days (Kuniyoshi et al. 2006a; Bower et al. 2007; Niinuma et al. 2007; Kida et al. 2008). We suggest that these radio transients may be generated by a single class of progenitors. The main characteristics of these "longduration radio transients" are (1) a very high occurrence rate of about $\sim 10^{3} \mathrm{deg}^{-2} \mathrm{yr}^{-1}$ in the $5 \mathrm{GHz}$ band, (2) common at intermediate Galactic latitude, with progenitor's sky surface density of $\gtrsim 60 \mathrm{deg}^{-2}$ at Galactic latitude, $b \sim 40^{\circ}$, (3) lacking any $\widetilde{X}$-ray $\left(\gtrsim 10^{-13} \mathrm{erg} \mathrm{cm}^{-2} \mathrm{~s}^{-1}\right)$, visible light $(g<27.6, R<26.5 \mathrm{mag})$, near-IR $(K<20.4 \mathrm{mag})$ and radio $\left(S_{5 \mathrm{GHz}} \gtrsim 10 \mu \mathrm{Jy}\right)$ counterparts, and (4) more abundant in the $5 \mathrm{GHz}$ band as compared to that in the $1.4 \mathrm{GHz}$ band. From the rates in the two bands, we infer the spectral index between the two bands is $\alpha \gtrsim 0$ where the flux density, $f_{v} \propto v^{\alpha}$.

These events are most probably not associated with the usual culprits like GRBs, SGRs, AGNs, SNe, flare stars, pulsars and interacting binaries (Section 5). We find that several other hypotheses including Galactic isolated old NSs; brown dwarfs and some sort of a new kind of explosion cannot be ruled out. Among these, we find that the association with isolated old NSs is especially attractive. We explore this hypothesis in detail and show that it is consistent with the current observations. In the framework of Galactic isolated old NSs, we show that (1) the typical distance to the Bower et al. sample (mJy events) is between $1 \mathrm{kpc}$ and $\sim 5 \mathrm{kpc}$, (2) the typical distance to the Kida et al. Jy-level events is less than about $2 \mathrm{kpc}$ and more than $45 \mathrm{pc}$ at the $95 \% \mathrm{CL}$, and (3) they will have a burst repetition timescale of about several months and duty cycle of $\sim 7 \times 10^{-3}$.

A possible association with isolated old NSs is exciting. If correct, this may prove to be the most practical way, so far, to find old NSs in our Galaxy and explore their demographics. Specifically, the demography of old NSs constitutes an excellent probe of the star formation history and metal enrichment of the Galaxy, and the gravitational potential of the Galaxy.

Our analysis naturally suggests several tests that can be used to rule out this hypothesis. First, if indeed long-duration radio transients are associated with isolated old NSs, then we expect them to be distributed inhomogeneously on the celestial sphere. Specifically, this hypothesis predicts that faint sub-mJy longduration radio transients will be at least a few times more common in the Galactic center than in high Galactic latitude. The exact ratio, however, depends on the distance scale to these events. This is illustrated in Figure 6 which shows the expected approximate distribution of isolated old NSs on the celestial sphere, for the entire NS population and NS which are at distance smaller than $1 \mathrm{kpc}$ from the Sun. Finally, we note that if the radio emission from such sources is pulsating, then pulsar searches conducted at $5 \mathrm{GHz}$ will find these objects. The fact that such "pulsars" were not found in existing surveys may be due to the fact that the majority of pulsar searches are carried on in low frequencies in which the rate of these transients is low.

We thank Re'em Sari, Orly Gnat, Ehud Nakar, Peter Goldreich, Stel Phinney, Dovi Poznanski, and Nat Butler for many discussions and for Robert Becker for valuable information regarding the strategy of the FIRST survey and information regarding VLA J172059.90+385226.6. Support for program number HST-GO-11104.01-A was provided by NASA through a grant from the Space Telescope Science Institute, which is operated by the Association of Universities for Research in Astronomy, Incorporated, under NASA contract NAS5-26555. A.G. acknowledges support by the Israeli Science Foundation, an EU Seventh Framework Programme Marie Curie IRG fellowship, and the Benoziyo Center for Astrophysics, a research grant from the Peter and Patricia Gruber Awards, and the William Z. and Eda Bess Novick New Scientists Fund at the Weizmann Institute.

\section{REFERENCES}

Agüeros, M. A., et al. 2006, AJ, 131, 1740

Arnett, W. D., Schramm, D. N., \& Truran, J. W. 1989, ApJ, 339, L25

Arons, J., \& Lea, S. M. 1976, ApJ, 207, 914

Arons, J., \& Lea, S. M. 1980, ApJ, 235, 1016

Arzoumanian, Z., Chernoff, D. F., \& Cordes, J. M. 2002, ApJ, 568, 289

Baraffe, I., Chabrier, G., Barman, T. S., Allard, F., \& Hauschildt, P. H. 2003, A\&A, 402, 701

Barbary, K., et al. 2009, ApJ, 690, 1358

Bastian, T. S., Benz, A. O., \& Gary, D. E. 1998, ARA\&A, 36, 131

Becker, R. H., White, R. L., \& Helfand, D. J. 1995, ApJ, 450, 559

Berger, E. 2002, ApJ, 572, 503

Berger, E. 2006, ApJ, 648, 629

Berger, E., et al. 2001, Nature, 410, 338 
Berger, E., et al. 2005, ApJ, 627, 960

Berger, E., et al. 2008a, ApJ, 673, 1080

Berger, E., et al. 2008b, ApJ, 676, 1307

Blaes, O., \& Madau, P. 1993, ApJ, 403, 690

Bondi, H., \& Hoyle, F. 1944, MNRAS, 104, 273

Bower, G. C., Saul, D., Bloom, J. S., Bolatto, A., Filippenko, A. V., Foley, R. J., \& Perley, D. 2007, ApJ, 666, 346

Brotherton, M. S., Tran, H. D., Becker, R. H., Gregg, M. D., LaurentMuehleisen, S. A., \& White, R. L. 2001, ApJ, 546, 775

Burgasser, A. J. 2004, ApJS, 155, 191

Burgasser, A. J., Kirkpatrick, J. D., Reid, I. N., Liebert, J., Gizis, J. E., \& Brown, M. E. 2000, AJ, 120, 473

Burgasser, A. J., \& Putman, M. E. 2005, ApJ, 626, 486

Burrows, A., et al. 1997, ApJ, 491, 856

Cameron, P. B., et al. 2005, Nature, 434, 1112

Camilo, F., Ransom, S. M., Halpern, J. P., Reynolds, J., Helfand, D. J., Zimmerman, N., \& Sarkissian, J. 2006, Nature, 442, 892

Carilli, C. L., Ivison, R. J., \& Frail, D. A. 2003, ApJ, 590, 192

Cenko, S. B., et al. 2008, ApJ, 677, 441

Chandra, P., et al. 2008, ApJ, 683, 924

Chanmugam, G. 1992, ARA\&A, 30, 143

Cole, S., et al. 2001, MNRAS, 326, 255

Colgate, S. A. 1975, ApJ, 198, 439

Colgate, S. A., \& Noerdlinger, P. D. 1971, ApJ, 165, 509

Colpi, M., Turolla, R., Zane, S., \& Treves, A. 1998, ApJ, 501, 252

Condon, J. J., Cotton, W. D., Greisen, E. W., Yin, Q. F., Perley, R. A., Taylor, G. B., \& Broderick, J. J. 1998, AJ, 115, 1693

Cordes, J. M., \& Chernoff, D. F. 1998, ApJ, 505, 315

Cordes, J. M., \& Shannon, R. M. 2008, ApJ, 682, 1152

DeBoer, D. R., et al. 2004, Proc. SPIE, 5489, 1021

Deneva, J. S., et al. 2009, ApJ, 703, 2259

Eichler, D. 2002, MNRAS, 335, 883

Falcke, H. D., et al. 2007, Highlights Astron., 14, 386

Faucher-Giguère, C.-A., \& Kaspi, V. M. 2006, ApJ, 643, 332

Fisher, N. I., Lewis, T., \& Embleton, B. J. J. 1987, Statistical Analysis of Spherical Data (Cambridge: Cambridge Univ. Press)

Frail, D. A., et al. 1997, ApJ, 483, L91

Fruchter, A. S., et al. 2006, Nature, 441, 463

Gal-Yam, A., et al. 2006, ApJ, 639, 331

Gehrels, N. 1986, ApJ, 303, 336

Gehrels, N., et al. 2004, ApJ, 611, 1005

Ghez, A. M., et al. 2008, ApJ, 689, 1044

Glikman, E., Helfand, D. J., \& White, R. L. 2006, ApJ, 640, 579

Gould, A., Bahcall, J. N., \& Flynn, C. 1996, ApJ, 465, 759

Gregg, M. D., Lacy, M., White, R. L., Glikman, E., Helfand, D., Becker, R. H., \& Brotherton, M. S. 2002, ApJ, 564, 133

Güdel, M. 2002, ARA\&A, 40, 217

Guetta, D., Piran, T., \& Waxman, E. 2005, ApJ, 619, 412

Haberl, F., Motch, C., \& Pietsch, W. 1998, Astron. Nachr., 319, 97

Hallinan, G., et al. 2007, ApJ, 663, L25

Harrison, F. A., et al. 1999, ApJ, 523, L121

Hawking, S. W. 1974, Nature, 248, 30

Helfand, D. J., Chanan, G. A., \& Novick, R. 1980, Nature, 283, 337

Hopkins, A. M., \& Beacom, J. F. 2006, ApJ, 651, 142

Hyman, S. D., Lazio, T. J. W., Kassim, N. E., Ray, P. S., Markwardt, C. B., \& Yusef-Zadeh, F. 2005, Nature, 434, 50

Hyman, S. D., Wijnands, R., Lazio, T. J. W., Pal, S., Starling, R., Kassim, N. E., \& Ray, P. S. 2009, ApJ, 696, 280

Johns-Krull, C. M., \& Valenti, J. A. 1996, ApJ, 459, L95

Jørgensen, P. S., Riis, T., Betto, M., \& Pickles, A. 2002, in ASP Conf. Ser. 281, Astronomical Data Analysis Software and Systems XI, ed. D. A. Bohlender, D. Durand, \& T. H. Handley (San Francisco, CA: ASP), 207

Kellermann, K. I., \& Pauliny-Toth, I. I. K. 1969, ApJ, 155, L71

Kida, S., et al. 2008, New Astron., 13, 519

Kinney, A. L., Calzetti, D., Bohlin, R. C., McQuade, K., Storchi-Bergmann, T., \& Schmitt, H. R. 1996, ApJ, 467, 38

Kramer, M., Lyne, A. G., O’Brien, J. T., Jordan, C. A., \& Lorimer, D. R. 2006, Science, 312, 549

Kulkarni, S. R., et al. 1998, Nature, 395, 663

Kuniyoshi, M., et al. 2006a, J. Phys. Conf. Ser., 31, 191
Kuniyoshi, M., et al. 2006b, PASP, 118, 901

Kuniyoshi, M., et al. 2007, PASP, 119, 122

Levinson, A., Ofek, E. O., Waxman, E., \& Gal-Yam, A. 2002, ApJ, 576, 923

Lorimer, D. R., Bailes, M., McLaughlin, M. A., Narkevic, D. J., \& Crawford, F. 2007, Science, 318, 777

Maoz, D., Ofek, E. O., \& Shemi, A. 1997, MNRAS, 287, 293

Matsumura, N., et al. 2009, AJ, 138, 787

McLaughlin, M. A., et al. 2006, Nature, 439, 817

Motch, C., Guillout, P., Haberl, F., Pakull, M., Pietsch, W., \& Reinsch, K. 1997, A\&A, 318, 111

Nakar, E., Gal-Yam, A., Piran, T., \& Fox, D. B. 2006, ApJ, 640, 849

Narayan, R., \& Ostriker, J. P. 1990, ApJ, 352, 222

Neuhäuser, R., \& Trümper, J. E. 1999, A\&A, 343, 151

Niinuma, K., et al. 2007, ApJ, 657, L37

Niinuma, K., et al. 2009, ApJ, 704, 652

Ofek, E. O. 2007, ApJ, 659, 339

Ofek, E. O. 2009, PASP, 121, 814

Ofek, E. O., et al. 2006, ApJ, 652, 507

Ofek, E. O., et al. 2008, ApJ, 681, 1464

Ostriker, J. P., Rees, M. J., \& Silk, J. 1970, ApL, 6, 179

Ovaldsen, J.-E., et al. 2007, ApJ, 662, 294

Perley, D. A., et al. 2009, AJ, 138, 1690

Perna, R., Narayan, R., Rybicki, G., Stella, L., \& Treves, A. 2003, ApJ, 594, 936

Peterson, B. M. 1997, An Introduction to Active Galactic Nuclei (Cambridge: Cambridge Univ. Press), 238

Phinney, E. S., \& Kulkarni, S. R. 1994, ARA\&A, 32, 591

Phinney, S., \& Taylor, J. H. 1979, Nature, 277, 117

Popov, S. B., Colpi, M., Treves, A., Turolla, R., Lipunov, V. M., \& Prokhorov, M. E. 2000, ApJ, 530, 896

Popov, S. B., \& Stern, B. E. 2006, MNRAS, 365, 885

Poznanski, D., Gal-Yam, A., Maoz, D., Filippenko, A. V., Leonard, D. C., \& Matheson, T. 2002, PASP, 114, 833

Press, W. H., Teukolsky, S. A., Vetterling, W. T., \& Flannery, B. P. 1992, Numerical Recipes (2nd ed.; Cambridge: Cambridge Univ. Press)

Quimby, R. M., et al. 2009, Nature, submitted (arXiv:0910.0059)

Readhead, A. C. S. 1994, ApJ, 426, 51

Rees, M. J. 1977, Nature, 266, 333

Reid, I. N., et al. 1999, ApJ, 521, 613

Reyes, R., et al. 2008, AJ, 136, 2373

Rutledge, R. E., Fox, D. W., Bogosavljevic, M., \& Mahabal, A. 2003, ApJ, 598, 458

Rybicki, G. B., \& Lightman, A. P. 1979, Radiative Processes in Astrophysics (New York: Wiley), 393

Sengupta, S. 1997, ApJ, 479, L133

Soderberg, A. M., et al. 2006, Nature, 442, 1014

Srinivasan, G. 1989, A\&AR, 1, 209

Sun, X., \& Han, J. 2002, Prog. Astron., 20, 130

Toropina, O. D., Romanova, M. M., Toropin, Y. M., \& Lovelace, R. V. E. 2001, ApJ, 561, 964

Toropina, O. D., Romanova, M. M., Toropin, Y. M., \& Lovelace, R. V. E. 2003, ApJ, 593, 472

Toropina, O. D., Romanova, M. M., Toropin, Y. M., \& Lovelace, R. V. E. 2005 , Mem. Soc. Astron. Ital., 76, 508

Treves, A., \& Colpi, M. 1991, A\&A, 241, 107

Treves, A., Colpi, M., \& Lipunov, V. M. 1993, A\&A, 269, 319

Treves, A., Popov, S. B., Colpi, M., Prokhorov, M. E., \& Turolla, R. 2001, in ASP Conf. Ser. 234, X-ray Astronomy 2000, ed. R. Giacconi, S. Serio, \& L. Stella (San Francisco, CA: ASP), 225

Urpin, V. A., \& Muslimov, A. G. 1992, MNRAS, 256, 261

Vachaspati, T. 2008, Phys. Rev. Lett., 101, 141301

Valenti, J. A., Marcy, G. W., \& Basri, G. 1995, ApJ, 439, 939

Voges, W., et al. 1999, A\&A, 349, 389

Weiler, K. W., van Dyk, S. D., Discenna, J. L., Panagia, N., \& Sramek, R. A. 1991, ApJ, 380, 161

White, R. L., Becker, R. H., Helfand, D. J., \& Gregg, M. D. 1997, ApJ, 475, 479

Yakovlev, D. G., \& Pethick, C. J. 2004, ARA\&A, 42, 169

Zhang, B., Gil, J., \& Dyks, J. 2007, MNRAS, 374, 1103 